\title{
A NEW SURVEY FOR GIANT ARCS
}

\author{
Joseph F. Hennawi ${ }^{1,14}$, Michael D. Gladders ${ }^{2,3,14}$, Masamune Oguri ${ }^{4,5}$, Neal Dalal ${ }^{6}$, Benjamin Koester ${ }^{7}$, \\ Priyamvada Natarajan ${ }^{8,9}$, Michael A. Strauss ${ }^{5}$, NaOhisa Inada ${ }^{10}$, Issha Kayo ${ }^{11}$, Huan Lin ${ }^{12}$, Hubert Lampeitl ${ }^{12}$, \\ James AnNis $^{12}$, Neta A. BAHCALL ${ }^{5}$, ANd Donald P. SChNeIDER ${ }^{13}$ \\ ${ }^{1}$ Department of Astronomy, University of California Berkeley, Berkeley, CA 94720, USA \\ ${ }^{2}$ Carnegie Observatories, Pasadena, CA 91101, USA \\ ${ }^{3}$ Department of Astronomy \& Astrophysics, University of Chicago, Chicago, IL 60637, USA \\ ${ }^{4}$ Kavli Institute for Particle Astrophysics and Cosmology, Stanford University, 2575 Sand Hill Road, Menlo Park, CA 94025, USA \\ 5 Princeton University Observatory, Princeton, NJ 08544, USA \\ ${ }^{6}$ Canadian Institute for Theoretical Astrophysics, University of Toronto, 60 St. George St., Toronto, Ontario, Canada M5S 3H8 \\ ${ }^{7}$ Physics Department, University of Michigan, Ann Arbor, MI 48109, USA \\ ${ }^{8}$ Department of Astronomy, Yale University, P.O. Box 208101, New Haven, CT 06511-8101, USA \\ ${ }^{9}$ Department of Physics, Yale University, P.O. Box 208120, New Haven, CT 06520-8120, USA \\ ${ }^{10}$ Institute of Astronomy, Faculty of Science, University of Tokyo, 2-21-1 Osawa, Mitaka, Tokyo 181-0015, Japan \\ ${ }^{11}$ Department of Physics and Astrophysics, Nagoya University, Chikusa-ku, Nagoya 464-8062, Japan \\ ${ }^{12}$ Fermi National Accelerator Laboratory, P.O. Box 500, Batavia, IL 60510, USA \\ ${ }^{13}$ Department of Astronomy and Astrophysics, Pennsylvania State University, 525 Davey Laboratory, University Park, PA 16802, USA \\ Received 2007 October 24; accepted 2007 October 29; published 2008 January 16
}

\begin{abstract}
We $\dot{\mathrm{s}} \dot{m}$ report on the first results of an imaging survey to detect strong gravitational lensing targeting the richest clusters selected from the photometric data of the Sloan Digital Sky Survey (SDSS) with follow-up deep imaging observations from the Wisconsin-Indiana-Yale NOAO (WIYN) $3.5 \mathrm{~m}$ telescope and the University of Hawaii 88 inch telescope (UH88). The clusters are selected from an area of $8000 \mathrm{deg}^{2}$ using the red cluster sequence technique and span the redshift range $0.1 \lesssim z \lesssim 0.6$, corresponding to a comoving cosmological volume of $\sim 2 \mathrm{Gpc}^{3}$. Our imaging survey thus targets a volume more than an order of magnitude larger than any previous search. A total of 240 clusters were imaged of which 141 had sub-arcsecond image quality. Our survey has uncovered 16 new lensing clusters with definite giant arcs, an additional 12 systems for which the lensing interpretation is very likely, and 9 possible lenses which contain shorter arclets or candidate arcs which are less certain and will require further observations to confirm their lensing origin. Among these new systems are several of the most dramatic examples of strong gravitational lensing ever discovered, with multiple bright arcs at large angular separation. These will likely become "poster-child" gravitational lenses similar to Abell 1689 and CL0024+1654. The new lenses discovered in this survey will enable future systematic studies of the statistics of strong lensing and their implications for cosmology and our structure formation paradigm.
\end{abstract}

Key words: cosmology: observations - dark matter - galaxies: clusters: general - gravitational lensing large-scale structure of universe - techniques: image processing

Online-only material: color figures

\section{INTRODUCTION}

Does the currently favored $\Lambda \mathrm{CDM}$ (Cold Dark Matter) cosmological model explain the detailed distribution of dark matter in galaxy clusters? Strong gravitational lensing by clusters is a powerful test of this model, probing the largest collapsed structures in the universe, where the density of dark matter is highest and where mass-to-light ratios are sufficiently high $(\sim 10-100)$ that baryons are unlikely to significantly influence the distribution of dark matter. The statistics of giant arcs-such as their number counts, redshift distribution (of both sources and lenses), the distribution of image separations, and the distribution of angles between arcs in multi-arc clustersprovide powerful constraints on the distribution of dark matter and its evolution over cosmic time. Detailed modeling of image positions in individual lens systems can constrain the mass profile of dark matter halos (e.g. Tyson et al. 1998; Smith et al. 2001; Sand et al. 2004; Broadhurst et al. 2005a), and even stronger constraints can be obtained when strongly lensed image positions are combined with larger-scale weak-lensing

14 Hubble Fellow. measurements (Smith et al. 2001; Kneib et al. 2003; Gavazzi et al. 2003; Broadhurst et al. 2005b; Oguri et al. 2005; Dalal et al. 2008, in preparation). Besides providing cosmological constraints, lensing clusters are natural gravitational telescopes, whose magnification facilitates the study of otherwise unobservable faint high-redshift background galaxies (Blain et al. 1999; Smail et al. 2002; Metcalfe et al. 2003; Santos et al. 2004).

Surveys for giant arcs in galaxy clusters began just a few years after they were discovered (Lynds \& Petrosian 1989). Smail et al. (1991) conducted the first homogenous arc survey by imaging 19 rich clusters in the $V$ band and identifying 20 candidate arcs and arclets. Luppino et al. (1999) conducted an imaging survey $(V<22)$ for giant arcs behind luminous $X$-ray clusters with $z>0.15$ and $L_{\mathrm{X}}>2 \times 10^{44} \mathrm{erg} \mathrm{s}^{-1}$, selected from the Extended Medium-Sensitivity Survey (EMSS), following the earlier EMSS imaging survey of Le Fevre et al. (1994). Luppino et al. found that 8 out of 38 clusters showed arcs with angular separation $\Delta \theta \gtrsim 10^{\prime \prime}$ from the brightest cluster galaxies (BCGs). The effective area of the Luppino et al. survey was $\sim 360 \mathrm{deg}^{2}$ (Dalal et al. 2004), corresponding to a giant arc abundance of one arc per $45 \mathrm{deg}^{2}$. Zaritsky \& Gonzalez (2003) discovered two arcs at small angular separations $\left(\Delta \theta \lesssim 10^{\prime \prime}\right)$ 
in deep imaging $(R<21.5)$ of 44 clusters in the redshift range $0.5<z<0.7$. These clusters comprised a subsample of the optically selected Las Campanas Distant Cluster Survey (LCDCS), and the effective area of their survey was $69 \mathrm{deg}^{2}$. Gladders et al. (2003) discovered eight lensing clusters in the $90 \mathrm{deg}^{2}$ imaging $\left(\mu_{\mathrm{R}}<24 \mathrm{mag} \operatorname{arcsec}^{-2}\right)$ area of the RedSequence Cluster Survey (RCS). Oddly, all eight of the Gladders et al. lensing clusters are at $z>0.64$ even though the RCS cluster catalog extends down to $z \sim 0.3$. Although many arc systems have been discovered from ground-based imaging, the superb image quality delivered by the Hubble Space Telescope $(H S T)\left(\mathrm{FWHM}=0.15^{\prime \prime}\right)$ enables the detection of much fainter lower surface brightness features which would be missed in ground-based images. Sand et al. (2005) exploited this fact, and mined the HST Wide Field and Planetary Camera 2 (WFPC2) data archive in the search for strong lensing. They presented $\sim 60$ arcs with image separations of $\Delta \theta \gtrsim 10^{\prime \prime}$, which lie in 32 of the 128 clusters they examined. ${ }^{15}$

Bartelmann et al. (1998) compared the rate of occurrence of giant arcs in a subsample of the EMSS (Le Fevre et al. 1994) to the theoretical predictions of ray tracing simulations through CDM clusters, and argued that the observed arc abundance exceeded the prediction of the $\Lambda \mathrm{CDM}$ cosmological model by an order of magnitude. More recent analyses of this claimed discrepancy have shown that $\Lambda \mathrm{CDM}$ is in reasonable agreement with giant arc statistics (Oguri et al. 2003; Wambsganss et al. 2004a; Dalal et al. 2004; Li et al. 2005; Horesh et al. 2005) at low redshift $(z<0.5)$. In particular, Dalal et al. (2004) argued that the order-of-magnitude discrepancy claimed by Bartelmann et al. (1998) was likely due to an underestimate of the EMSS survey volume, an overestimate of the density of background sources, and a slight overestimate of the lensing optical depth. Hennawi et al. (2007b) recently compared the abundance of giant arcs in the Gladders et al. (2003) RCS sample to predictions from ray tracing simulations of $\Lambda \mathrm{CDM}$, and found good agreement for the number of $z>0.6$ lenses; however, a similar number of $z<0.6$ lenses were predicted in the RCS area which were not observed. Although this putative discrepancy is based on only a handful of objects, it should be emphasized that the Hennawi et al. (2007b) study used $\sigma_{8}=0.95$, which is discrepant at the $4 \sigma$ level with the lower most recent WMAP measurement of $\sigma_{8}=0.76 \pm 0.05$ (Spergel et al. 2006), and is $1.5 \sigma$ discrepant with the measurement from the RCS cluster abundance of $\sigma_{8}=0.67_{-0.13}^{+0.18}$ (Gladders et al. 2006). A lower value of $\sigma_{8}$ would significantly reduce the predicted number of high-redshift clusters, making the RCS results even more discrepant with theoretical predictions ( $\mathrm{Li}$ et al. 2006). This putative excess of high-redshift lensing clusters, as well as the observation that the proportion of lensing clusters with arcs from multiple background sources is very high $(\sim 50 \%)$ (but see Ho \& White 2005), led Gladders et al. (2003) to speculate that the cluster lensing at high redshift is caused by a sub-population of cluster "superlenses," which have much larger lensing cross section than the cluster population as a whole.

To date, the three best-studied strong lensing clusters are Abell 1689, CL0024+1654, and MS2137.3-2353. The presence of multiple-lensed arcs in these systems has enabled detailed modeling of image positions which strongly constrains the distribution of dark matter, especially when combined with larger-scale weak-lensing measurements. Recent analyses of

\footnotetext{
15 For ease of comparison with previous surveys we quote the length-to-width ratios for arcs with $L / W>10$, whereas Sand et al. published all arcs with $L / W>7$.
}

the mass profiles in these clusters (Gavazzi et al. 2003; Kneib et al. 2003; Broadhurst et al. 2005a) have uncovered highly concentrated mass distributions. All three clusters have primary mass components with NFW concentrations $c_{\mathrm{NFW}}>14$, while Hennawi et al. (2007b) showed that such high concentrations are expected in $<2 \%$ of clusters which show giant arcs (see also Oguri et al. 2005). Why should the three best-studied lensing clusters in the universe all lie on the tail of the concentration distribution? Is this merely a selection bias or does it point to a deeper problem with the $\Lambda \mathrm{CDM}$ model on cluster scales? A larger sample of similar multi-arc lensing clusters will be required to determine if the concentrations of cluster lenses are really discrepant with $\mathrm{CDM}$, which brings us to the subject of this work.

In this paper we describe a survey for lensing clusters which aims to improve the statistics of lensing clusters by exploiting the $\gtrsim 1 \mathrm{Gpc}^{3}$ cosmological volume of the Sloan Digital Sky Survey (SDSS). We have carried out a deep imaging $\left(\mu_{g} \lesssim 25.7\right)$ search for giant arcs, conducted on the Wisconsin-Indiana-Yale NOAO (WIYN) $4 \mathrm{~m}$ telescope and the University of Hawaii 88 inch telescope (UH88), which targets the richest clusters identified in the SDSS photometric data. The red cluster sequence algorithm (Gladders \& Yee 2000, 2005) was applied to the $\sim 8000 \mathrm{deg}^{2}$ of SDSS photometric data, resulting in the largest catalog of massive galaxy clusters in existence (Gladders et al. 2008a, in preparation); our giant arc survey covers an area 20 times larger than any previous search for cluster lensing.

The input cluster catalog to our survey is briefly described in Section 2 . The deep imaging observations and data reduction are discussed in Section 3. We explain how we identified giant arcs and present our sample of new lensing clusters in Section 4. Notes on individual systems are given in Section 5, and we summarize and conclude in Section 6. Throughout this work we use the best fit three-year WMAP only (maximum likelihood) cosmological model of Spergel et al. (2006), which has $\Omega_{m}=$ $0.24, \Omega_{\Lambda}=0.76, h=0.73$.

\section{CLUSTER SELECTION}

The SDSS (York et al. 2000) uses a dedicated $2.5 \mathrm{~m}$ telescope and a large format CCD camera (Gunn et al. 1998, 2006; Tucker et al. 2006) at the Apache Point Observatory in New Mexico to obtain images in five broad bands $(u, g, r, i$, and $z$, centered at $3551,4686,6166,7480$, and $8932 \AA$, respectively; Fukugita et al. 1996; Stoughton et al. 2002) of high Galactic latitude sky in the Northern Galactic Cap. The imaging data are processed by the astrometric pipeline (Pier et al. 2003) and photometric pipeline (Lupton et al. 2001), and are photometrically calibrated to a standard star network (Hogg et al. 2001; Smith et al. 2002; Ivezić et al. 2004). Based on these imaging data, spectroscopic targets chosen by various selection algorithms (i.e., quasars, galaxies, stars, serendipity) are observed with two double spectrographs producing spectra covering 3800-9200 $\AA$ with a spectral resolution ranging from 1800 to $2100\left(\mathrm{FWHM} \simeq 150-170 \mathrm{~km} \mathrm{~s}^{-1}\right)$. Details of the spectroscopic observations can be found in Castander et al. (2001) and Stoughton et al. (2002). Additional details on the SDSS data products can be found in Abazajian et al. (2003, 2004, 2005) and Adelman-McCarthy (2006).

The total imaging area of the SDSS Data Release 5 (DR5, Adelman-McCarthy 2008, in preparation) is $\sim 8000 \mathrm{deg}^{2}$. We applied the RCS selection algorithm (Gladders \& Yee 2000, 2005 ) to the SDSS photometric galaxy catalog, and produced a 
sample of $\sim 2 \times 10^{4}$ massive galaxy clusters over the redshift range $0.05 \lesssim z \lesssim 0.6$, which is the largest catalog of massive clusters in existence. The RCS algorithm exploits the fact that all clusters have a red sequence of early-type galaxies, and a cluster is localized as an overdensity in position, magnitude, and color simultaneously. This method has been demonstrated to be robust and effective in the RCS survey (Gladders \& Yee 2000, 2005), and has been tested on both real and mock data. Using photometric redshift techniques, the RCS algorithm yields cluster redshifts which are accurate to $\lesssim 0.05$ by fitting for the location of the red sequence of the cluster galaxies in the colormagnitude plane. These initial approximate redshifts were used as the input to our follow-up imaging survey. For those clusters which we imaged at WIYN or UH88, we re-computed more accurate 5-band photometric redshifts $\sigma \lesssim 0.02$, restricting attention to only those galaxies which satisfied the Luminous Red Galaxy (LRG) color-selection criteria (Eisenstein et al. 2001; Padmanabhan et al. 2005), because these galaxies have been demonstrated to have more robust photometric redshifts, accurate to $\sigma_{z} \sim 0.03$ per galaxy in the redshift range $0.2 \lesssim z \lesssim$ 0.55 . We employed the photometric redshift algorithm described in Padmanabhan et al. (2005).

Only clusters with redshifts $z \gtrsim 0.1$ were targeted for followup imaging, since strong lensing is significantly less likely behind lower-redshift clusters (see, e.g., Hennawi et al. 2007b). The cosmological volume accessible to our survey is thus $1.9 h^{-1} \mathrm{Gpc}^{3}\left(0.1 \lesssim z \lesssim 0.6 ; \sim 8000 \mathrm{deg}^{2}\right)$. The clusters were assigned priorities based on a combination of richness, detection significance, and a parameter that describes the concentration of the galaxy distribution, with the richest, most significant, most highly concentrated clusters receiving a higher priority. The redshift range $z=0.1-0.6$ was divided into five equal bins of width $d z=0.1$, priorities were assigned based on the relative rank of the cluster in that bin, and higher priority clusters were targeted first. We adopted the strategy of ranking the clusters in redshift bins for two reasons. First, the quantities such as richness and detection significance which were used to rank the clusters are strong functions of redshift, so that a comparison of these quantities between two clusters with very different redshifts was not sensible. Second, one of the goals of our survey is to understand the redshift distribution of cluster lenses and hence uniform sampling of the accessible redshift range was desired. Previously known lensing clusters were not excluded from our imaging survey, although we were somewhat less likely to observe them. Also, we were less likely to observe clusters in the lowest redshift bin $0.1<z<0.2$ because of the lower efficiency for strong lensing for low-redshift clusters.

We note that the cluster catalog was in a preliminary state at the time of the observations, and the criteria used to rank the clusters or follow-up imaging varied somewhat from run to run. In light of this, our imaging survey suffers from inhomogeneous selection which will be remedied as the survey is completed. Full details of the SDSS-RCS cluster catalog (Gladders et al. 2008a, in preparation) and the selection criteria for our cluster lens survey (Gladders et al. 2008b, in preparation) will be presented in future papers.

In addition to the clusters selected by the RCS algorithm, we also imaged a handful of candidates for which visual inspection of the SDSS imaging suggested strong lensing. One of these objects is the lensing cluster SDSS J0146-0929, which was serendipitously discovered in the SDSS imaging by Pat Hall (Estrada et al. 2007). The others were candidate cluster lenses identified via visual inspection of the SDSS co-added southern strip imaging (Estrada et al. 2007), for which the lensing interpretation was ambiguous because the SDSS imaging was too shallow.

\section{DEEP IMAGING SURVEY}

\subsection{WIYN 3.5 Imaging}

The WIYN 3.5 m telescope imaging observations took place during three runs on 2004 June 10-12, 2005 May 7-12, and 2006 May 24-27, for which conditions were photometric. An additional run on 2006 June 27-28 was completely lost to poor weather. For the 2005 and 2006 runs, we used the Orthogonal Parallel Transfer Imaging Camera (OPTIC; Howell et al. 2003), which consists of two Lincoln Lab CCID28 $2 \mathrm{~K} \times 4 \mathrm{~K}$ chips, resulting in a $4 \mathrm{~K} \times 4 \mathrm{~K}$ imager. For the 10-12 June 2004 run, we used the WIYN Mini-Mosaic Imager (Minimo; Saha et al. 2000 ), which is also a $4 \mathrm{~K} \times 4 \mathrm{~K}$ imager, but consisting of two SITe $4096 \times 2048$ CCDs. Both OPTIC and Minimo have $15 \mu \mathrm{m}$ pixels subtending $0.14^{\prime \prime}$, giving rise to a total field of view of $9.6 \times 9.6$ arcmin. The readout time for OPTIC is $25 \mathrm{~s}$, whereas Minimo takes $182 \mathrm{~s}$ to read out the full frame. The combined median seeing of all of our WIYN runs was $0.90^{\prime \prime}$, measured in the $g$ bandpass.

We observed each cluster for $600 \mathrm{~s}$, broken into two $300 \mathrm{~s}$ exposures. The majority of the clusters were observed in this mode. We chose the SDSS $g$ bandpass both because giant arcs are known to typically be blue and to exploit the fact that the night sky is darker in $g$ than in a redder bandpass. The 2004 June 10-12 imaging using Minimo was also conducted in $g$; however, we performed longer, $2400 \mathrm{~s}$, integrations. We chose longer, $600 \mathrm{~s}$, individual exposures with Minimo because of the considerable readout time (182 s) of this detector.

Each image was bias subtracted using the overscan region and median bias frames, and flat-fielded using a median twilight sky flat. Final images were produced by registering and shifting the images, and cleaning them of cosmic ray hits.

\subsection{UH 88 Inch Imaging}

The UH 88 inch telescope was employed during two runs on 2005 May 6-7 and 2006 April 24-25, using the University of Hawaii 8k wide field imager (UH8k), which is a mosaic CCD camera consisting of eight $2048 \times 4096$ pixel CCDs, resulting in a total of $8192 \times 8192$ pixels. The plate scale is $0.235^{\prime \prime}$, giving rise to a $32 \times 32$ arcmin field of view. The median seeing for the 2005 May and 2006 April run was $0.86^{\prime \prime}$ and $1.13^{\prime \prime}$, respectively, measured in the $V$ bandpass. Conditions were not photometric for the April 2006 run. One of our serendipitous lensing cluster targets (SDSS J0146-0922; see Section 4) was observed on 2004 December 17 as part of a different observing program.

Our observing strategy at the UH 88 inch differed for the two runs. During the 2005 May 6-7 run, we imaged clusters in the $V$ bandpass for total integration times of $1800 \mathrm{~s}$. The total integrations were shortened to $900 \mathrm{~s}$ for the run of 2006 April 24-25. The $V$ band was chosen because this was the bluest filter available for the UH8k camera at the time of observing. The individual exposure times for the UH8k were $180 \mathrm{~s}$, which were chosen because guiding is not available for this instrument, and longer integrations would have resulted in image distortions.

Each image was bias subtracted using a median bias frame, and flat-fielded using a median dome flat. Final images were produced by registering and shifting the images, and averaging the individual exposures. 


\subsection{Photometric Calibration and Seeing Measurements}

Astrometric solutions were obtained for our images using the SDSS astrometry of stars identified in each image. Images were then photometrically calibrated to the SDSS photometry using stars and galaxies. Because the UH88 images were taken in the $V$ band, the photometric transformations given in Fukugita et al. (1996) were used to produce estimated $V$-band magnitudes for SDSS objects within these images, and hence a nominal $V$-band calibration. For the WIYN/OPTIC data (600 s integrations), our $1 \sigma$ surface brightness limit in a $1^{\prime \prime}$ aperture is typically $\mu_{g}=25.7 \pm 0.1$, whereas the deeper (2400 s) WIYN/Minimo images from the 2004 June 10-12 run have a fainter limit of $\mu_{g}=26.5 \pm 0.1$. For the UH88 images from the 2005 May 6-7 (1800 s exposures) the surface brightness limit was $\mu_{V}=26.9 \pm 0.1$. Seeing was measured for each image by fitting a Gaussian PSF to several stars in each image.

\section{LENSING CLUSTER SAMPLE}

The final best-seeing image for each cluster was visually inspected by two of the authors (J. Hennawi and M. Gladders) independently. Possible lensing features were assigned an integer grade of 1-3 (1 being "possible," 2 "likely," and 3 "definite"), and pixel positions of each feature were noted. The intent of these rankings is such that a well-detected giant arc with appropriate curvature and orientation would receive a grade of 3 , putative arclets would receive a grade of 1 , and grade 2 features typically are apparent giant arcs at the sky limit, or brighter apparent arcs with less plausible or ambiguous image geometries, etc. The complete lists of each examiner were then matched within a radius of $10^{\prime \prime}$, and the rankings summed, producing a final candidate list of lensing features with rankings between 1 and 6 . We report in this paper on three sub-samples derived from this list. The first and most definite sample contains objects with a total rank of 4 or greater (which at the lower limit were either assigned a grade of 3 by at least one of the examiners, or more typically a 2 by both examiners). This is the core sample of the paper which we refer to as our "definite" lensing cluster sample. These represent the clusters for which we have the greatest confidence in a strong lensing interpretation. Next are all objects with a total rank equal to 3 , which we consider as "likely" strong lensing features. Our final sample of "possible" cluster lenses, consists of objects which received a score of 2 from one examiner but were missed by the other. These features were typically missed by one of us because they are short arcs/arclets at the sky limit or they were brighter features but were located well away from the cluster center. These possible lensing clusters will require further imaging or spectroscopic observations to confirm their lensing origin.

The three samples are summarized in Tables 1-3, which give coordinates and redshifts for the clusters, the seeing of the arc discovery image, the telescope and instrument used for the observation, and brief notes on the lensed features and clusters. Cutout images of the clusters in the respective samples are shown in Figures 3-5. Strong lensing features are indicated by lines and a number which is the total score of the identified strong lensing feature.

The cluster redshifts are derived either from SDSS spectroscopy, from a photometric redshift analysis of the SDSS photometry of cluster members, or for previously known clusters which did not have an SDSS spectroscopic redshift, from the literature. For clusters with SDSS spectra, the redshift reported is that of the early-type galaxy nearest to the cluster center

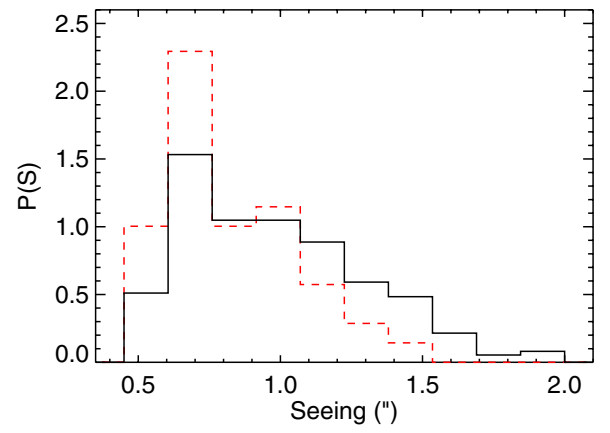

Figure 1. Seeing distributions for the clusters imaged in our survey. The solid (black) histogram shows the seeing distribution for the 240 clusters imaged in our survey, while the dashed (red) histogram is the seeing distribution for the 45 clusters which were identified as candidate strong lenses (see Section 4). We imaged 141 clusters in sub-arcsecond seeing; the median seeing for the total survey is $0.94^{\prime \prime}$, and 141 clusters were imaged in sub-arcsecond seeing. The median seeing for the lenses was $0.74^{\prime \prime}$.

(A color version of this figure is available in the online journal)

which had a redshift consistent with the photometrically derived cluster redshift. For the photometric redshifts, we examined the photo- $z$ distribution of all galaxies within a projected aperture of $0.5 h^{-1} \mathrm{Mpc}$ from the cluster center which also satisfied the LRG color-cuts of Padmanabhan et al. (2005), which are based on the initial SDSS LRG color selection described in Eisenstein et al. (2001). We extended the LRG flux limits 0.7 magnitudes fainter than those used by Padmanabhan et al. (2005) to obtain more LRG cluster members and better statistics on the cluster redshift. This amounted to a flux limit of $r<20.4$ for LRGs in the redshift range $0.2 \lesssim z \lesssim 0.4$, and $i<20.7$ in the redshift range $0.4 \lesssim z \lesssim 0.6$ (see Padmanabhan et al. 2005 for details). We took the cluster redshift distribution to be the sum of a set of Gaussians with means and dispersions given by the photo- $z$ and the photo- $z$ error of the individual galaxies. The mode of this distribution was then taken to be the cluster photometric redshift. The average number of LRGs within the $0.5 h^{-1} \mathrm{Mpc}$ aperture was 14 for the 240 clusters which we imaged. Of these, $65 \%$ had SDSS spectroscopic redshifts, for which the average difference between the spectroscopic redshift and the cluster photometric redshift was $\left\langle\left|z_{\text {phot }}-z_{\text {spec }}\right|\right\rangle=0.019$, and the median of the same quantity was 0.013 .

In summary, a total of 240 clusters were imaged (195 from WIYN and 45 from UH88), out of which we found 22 definite cluster lenses (6 previously known), 14 likely lenses ( 2 previously known), and 9 possible systems. Because our imaging runs have only been in the Spring months of AprilJune, we have primarily covered the range of R.A. $=10-17 \mathrm{~h}$, and our survey of high priority clusters is roughly half-finished, so that a comparable number of systems are to be expected after the completion of the survey. The solid (black) curve in Figure 1 shows the seeing distribution for our imaging survey and the dashed (red) curve is the seeing distribution for the 45 clusters listed in Tables 1-3. The median seeing of all of our imaging is $0.94^{\prime \prime}$, while for the lenses it is $0.74^{\prime \prime}$, illustrating that we were more likely to identity lensing features with better image quality. Figure 2 compares the redshift distribution of the clusters we imaged (solid, black) to that of the lenses (dashed, red). The cutoff in the histograms for $z \lesssim 0.2$ reflects the fact that we were less likely to observe lower redshift clusters. At $z \gtrsim 0.5$, only the richest massive clusters are being identified in the SDSS photometric survey, which explains the high redshift cutoff (see Gladders et al. 2008a, in preparation). 
Table 1

Parameters for Definite Lensing Clusters

\begin{tabular}{|c|c|c|c|c|c|c|}
\hline Cluster name & $\begin{array}{l}\text { R.A. } \\
\text { (2000) }\end{array}$ & $\begin{array}{l}\text { Decl. } \\
(2000)\end{array}$ & $z$ & $\begin{array}{c}\text { Seeing } \\
\left({ }^{\prime \prime}\right)\end{array}$ & $\begin{array}{l}\text { Telescope/ } \\
\text { Instrument }\end{array}$ & Notes \\
\hline SDSS J0146-0929 & $01: 46: 56.01$ & $-09: 29: 52.5$ & 0.444 & 0.97 & UH88/8k & Known lens \\
\hline SDSS J1115+5319 & $11: 15: 14.85$ & $+53: 19: 54.6$ & 0.466 & 1.02 & WIYN/OPTIC & Complex morphology \\
\hline NSC J115347+425155 & $11: 53: 49.49$ & $+42: 50: 43.1$ & 0.327 & 0.58 & WIYN/OPTIC & Arc near $\mathrm{cD}$ \\
\hline SDSS J1217+3641 & $12: 17: 31.94$ & $+36: 41: 12.3$ & 0.364 & 0.71 & WIYN/OPTIC & Confusion from nearby galaxy \\
\hline Abell 1703 & $13: 15: 05.24$ & $+51: 49: 02.6$ & 0.281 & 0.97 & WIYN/Minimo & Two arcs \\
\hline GHO $132029+315500$ & $13: 22: 48.77$ & $+31: 39: 17.8$ & 0.307 & 1.00 & WIYN/OPTIC & Definite giant arc \\
\hline RX J1327.0+0211 & $13: 27: 01.01$ & $+02: 12: 19.5$ & 0.260 & 0.64 & WIYN/OPTIC & High surface-brightness arc \\
\hline NSC J134610+030555 & $13: 46: 03.53$ & $+03: 09: 31.0$ & 0.232 & 0.66 & WIYN/OPTIC & Faint arc \\
\hline Abell 1835 & 14:01:02.07 & $+02: 52: 42.5$ & 0.252 & 1.06 & WIYN/Minimo & Known lens \\
\hline Abell 1914 & $14: 25: 56.67$ & $+37: 48: 59.3$ & 0.170 & 0.62 & WIYN/OPTIC & Known lens \\
\hline Abell 1942 & $14: 38: 21.87$ & $+03: 40: 13.2$ & 0.225 & 0.79 & WIYN/OPTIC & Known lens \\
\hline SDSS J1446+3032 & $14: 46: 34.02$ & $+30: 32: 58.2$ & 0.47 & 0.63 & WIYN/OPTIC & Multiple bright arcs \\
\hline Abell 2070 & $15: 24: 19.51$ & $+35: 15: 59.3$ & 0.253 & 0.80 & WIYN/OPTIC & Definite bright arc \\
\hline SDSS J1531+3414 & $15: 31: 10.60$ & $+34: 14: 25.0$ & 0.335 & 1.20 & WIYN/OPTIC & Multiple high surface brightness arcs \\
\hline Abell 2141 & $15: 57: 42.40$ & $+35: 30: 29.8$ & 0.159 & 0.63 & WIYN/OPTIC & Bright arc with knots. \\
\hline SDSS J1557+2131 & $15: 57: 44.84$ & $+21: 31: 49.3$ & 0.427 & 0.54 & WIYN/OPTIC & CB58's? \\
\hline SDSSJ1602+4038 & $16: 02: 24.68$ & $+40: 38: 50.1$ & 0.387 & 0.58 & WIYN/OPTIC & Length/center uncertain \\
\hline Abell 2219 & $16: 40: 19.81$ & $+46: 42: 41.5$ & 0.234 & 0.69 & WIYN/OPTIC & Known lens \\
\hline RX J1652.2+4449a & $16: 52: 14.59$ & $+44: 49: 23.9$ & 0.175 & 0.67 & WIYN/OPTIC & Arc near BCG \\
\hline Abell $2261^{\mathrm{b}}$ & $17: 22: 27.21$ & $+32: 07: 55.1$ & 0.224 & 0.66 & WIYN/OPTIC & Known lens \\
\hline SDSS J1747+5428 & $17: 47: 21.76$ & $+54: 28: 13.9$ & 0.31 & 1.01 & WIYN/OPTIC & Arc near BCG \\
\hline SDSS J2111-0115 & $21: 11: 19.34$ & $-01: 14: 23.5$ & 0.68 & 0.74 & WIYN/Minimo & Multiple high surface brightness arcs \\
\hline
\end{tabular}

Notes. Parameters for definite lensing clusters shown in Figure 3. The coordinates are those of the brightest cluster galaxy as identified by our cluster finding algorithm. For cases where the arcs surround a secondary concentration of galaxies, the coordinates of a cluster member in this concentration are substituted. Spectroscopic redshifts, either from the SDSS or from the literature, are quoted to three significant digits, while photometric cluster redshift are quoted to two digits. The seeing of the arc discovery image is indicated, as well as the telescope and instrument used. Brief notes about the lensing features for each clusters are included. For more detailed notes including the references for known lenses see Section 5. Clusters which are labeled as SDSS are new discoveries in the RCS cluster catalog (Gladders et al. 2006). Previously known clusters are indicated by their names. Abell clusters are from the compilation of Abell et al. (1989). Clusters labeled NSC were previously discovered in the optical catalog of Gal et al. (2003). GHO indicates clusters from the photographic distant survey of Gunn et al. (1986). Clusters labeled RX are X-ray clusters from the NORAS catalog of Böhringer et al. (2000)

${ }^{a}$ Spectroscopic redshift from the NORAS catalog (Böhringer et al. 2000).

b Spectroscopic redshift from Crawford et al. (1995).

Table 2

Parameters for Tentative Lensing Clusters

\begin{tabular}{lcccccl}
\hline \hline Cluster name & $\begin{array}{c}\text { R.A. } \\
(2000)\end{array}$ & $\begin{array}{c}\text { Decl. } \\
(2000)\end{array}$ & $z$ & $\begin{array}{c}\text { Seeing } \\
\left({ }^{\prime \prime}\right)\end{array}$ & $\begin{array}{l}\text { Telescope/ } \\
\text { Instrument }\end{array}$ & \multicolumn{1}{c}{ Notes } \\
\hline SDSS J1040+3405 & $10: 40: 19.72$ & $+34: 05: 19.8$ & 0.445 & 1.10 & UH88/8k & Short arc \\
SDSS J1131+3554 & $11: 31: 10.08$ & $+35: 54: 19.0$ & 0.49 & 1.10 & WIYN/OPTIC & Confusion from bright star \\
Abell 1351 & $11: 42: 23.02$ & $+58: 30: 45.0$ & 0.322 & 0.97 & UH88/8k & Known lens \\
SDSS J1150+0650 & $11: 50: 30.29$ & $+06: 50: 19.1$ & 0.301 & 1.11 & WIYN/OPTIC & Arclet \\
Abell 1550 & $12: 29: 02.53$ & $+47: 36: 56.0$ & 0.262 & 1.30 & WIYN/OPTIC & Faint arc \\
SDSS J1258+4702 & $12: 58: 02.09$ & $+47: 02: 54.2$ & 0.32 & 1.39 & WIYN/OPTIC & Arc near BCG \\
Abell 1758 & $13: 32: 38.42$ & $+50: 33: 35.7$ & 0.279 & 0.85 & WIYN/OPTIC & Known lens \\
SDSS J1406+3945 & $14: 06: 32.72$ & $+39: 45: 46.2$ & 0.427 & 0.89 & UH88/8k & Short arc \\
SDSS J1414+2703 & $14: 14: 39.13$ & $+27: 03: 10.5$ & 0.46 & 0.63 & WIYN/OPTIC & Many suggestive features \\
SDSS J1527+0652 & $15: 27: 45.83$ & $+06: 52: 33.6$ & 0.40 & 0.67 & WIYN/OPTIC & Very high surface brightness \\
SDSS J1537+3926 & $15: 37: 52.22$ & $+39: 26: 09.9$ & 0.444 & 0.57 & WIYN/OPTIC & Faint resolved arc \\
Abell 2136 & $15: 53: 18.86$ & $+51: 07: 24.9$ & 0.229 & 0.87 & WIYN/Minimo & Multiple arclets \\
GHO 155414+405306 & $15: 55: 57.95$ & $+40: 44: 14.8$ & 0.393 & 0.52 & WIYN/OPTIC & Ambiguous \\
RXC J1749.3+4245 & $17: 49: 18.05$ & $+42: 46: 38.4$ & 0.230 & 0.63 & WIYN/OPTIC & Short blobby arc \\
\hline
\end{tabular}

Notes. Same as Table 1, but for the tentative lensing clusters shown in Figure 4.

a Spectroscopic redshift from the NORAS catalog (Böhringer et al. 2000).

\section{NOTES ON CLUSTER LENSES}

In this section we briefly describe the cluster lenses published in Tables 1-3. Each cluster in the definite and likely samples is discussed, as well as a few of the more notable clusters in the possible lens sample. We also discuss previously known lenses which we re-discovered in our search, as well as known lensing clusters which were imaged but whose lensing features were missed. We matched all of the clusters discussed below to the Northern ROSAT All-Sky (NORAS) galaxy cluster sample 
Table 3

Parameters for Possible Lensing Clusters

\begin{tabular}{lccccll}
\hline \hline Cluster name & $\begin{array}{c}\text { R.A. } \\
(2000)\end{array}$ & $\begin{array}{c}\text { Decl. } \\
(2000)\end{array}$ & $z$ & $\begin{array}{c}\text { Seeing } \\
\left({ }^{\prime \prime}\right)\end{array}$ & $\begin{array}{c}\text { Telescope/ } \\
\text { Instrument }\end{array}$ & \multicolumn{1}{c}{ Notes } \\
\hline SDSS J0821+2519 & $08: 21: 21.27$ & $+25: 19: 03.0$ & 0.268 & 1.05 & UH88/8k & Possible faint arc \\
SDSS J1008+4529 & $10: 08: 54.85$ & $+45: 29: 13.2$ & 0.479 & 0.81 & UH88/8k & Possible pair of faint pair \\
NSC J113554+40012 & $11: 35: 59.06$ & $+40: 05: 17.9$ & 0.295 & 0.88 & UH88/8k & Possible faint arc \\
SDSS J1240+4250 & $12: 40: 29.98$ & $+42: 50: 08.1$ & 0.406 & 0.73 & WIYN/OPTIC & Geometry ambiguous \\
Abell 1926 & $14: 30: 28.62$ & $+24: 40: 19.3$ & 0.135 & 1.33 & WIYN/OPTIC & Arclet? \\
Abell 1934 & $14: 33: 21.85$ & $+29: 27: 01.3$ & 0.220 & 0.63 & WIYN/OPTIC & Possible faint arc \\
SDSSJ1513+0525 & $15: 13: 46.27$ & $+05: 25: 38.6$ & 0.49 & 0.69 & WIYN/OPTIC & High SB images at large separation \\
NSC J163346+243312 & $16: 33: 48.85$ & $+24: 32: 37.3$ & 0.195 & 0.54 & WIYN/OPTIC & Faint arclets around secondary group \\
Abel1 2224 & $16: 43: 28.83$ & $+13: 21: 53.9$ & 0.126 & 0.50 & WIYN/OPTIC & Pair of arclets around secondary group \\
\hline
\end{tabular}

Notes. Same as Table 1, but for the possible lensing clusters shown in Figure 5.

${ }^{a}$ Spectroscopic redshift from Struble \& Rood (1987).

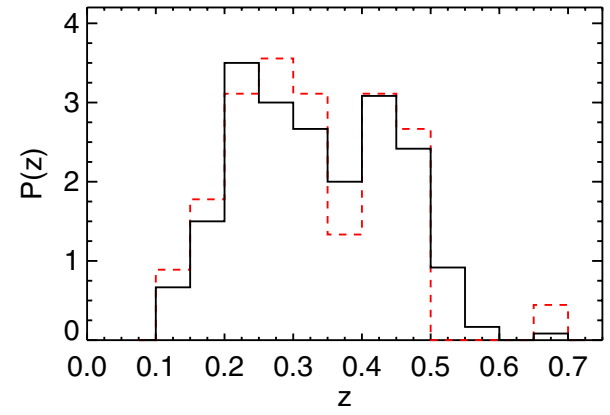

Figure 2. Redshift distributions of the clusters imaged in our survey. The solid (black) histogram is the redshift distribution of the 240 clusters imaged in our survey, while the dashed (red) histogram is the seeing distribution for the 45 clusters which were identified as candidate strong lenses (see Section 4).

(A color version of this figure is available in the online journal)

(Böhringer et al. 2000), the ROSAT-ESO Flux Limited X-ray (REFLEX) galaxy cluster sample (Böhringer et al. 2004), and also the ROSAT All Sky Survey-SDSS cluster sample (RASSSDSS; Popesso et al. 2004). For the clusters detected, we note $\mathrm{X}$-ray luminosities in the $0.1-2.4 \mathrm{keV}$ energy band, converted to the $\Lambda \mathrm{CDM}$ cosmology used in this paper.

\subsection{Definite Lenses}

SDSSJ 0146-0929. This lens at $z=0.444$ was serendipitously discovered in the SDSS imaging by Pat Hall (Estrada et al. 2007). Our UH88/8k $V$-band image shows three high surface brightness arcs at $\Delta \theta \sim 13^{\prime \prime}$ from the central galaxy. The morphology of the arcs is that of a classic quad configuration, which suggests that these are three images of the same multiply imaged source, as does the comparable surface brightness and similar colors of the images.

SDSSJ 1115+5319. Three candidate arcs were identified in our WIYN image (seeing $=11^{\prime \prime} 02$ ) of this cluster at $z=0.466$, which is one of the richest $z>0.4$ clusters in our catalog. The most compelling feature is the arc situated $\Delta \theta=31^{\prime \prime}$ east of the BCG (see Figure 3), which received a score of 6 . This arc threads between two cluster members, suggesting that the galaxies help in boosting the lensing potential. Two other arcs were identified situated to the southeast at $\Delta \theta=35^{\prime \prime}$ and $\Delta \theta=57^{\prime \prime}$, and received scores of 3 and 4 , respectively. The furthest of these two arcs also flanks a cluster member. The morphology of the cluster is very irregular with the galaxy distribution highly elongated along the east-west direction.
NSC J115347+425155. This cluster at $z=0.327$ has a complex morphology. There are several bright early type galaxies at the same redshift with comparable magnitudes, making the determination of the cluster center somewhat arbitrary. One of these secondary mass concentrations extends as far as $\sim 2.5^{\prime}$ to the north of the putative cluster center. Three candidate strong lensing features are identified, two of which straddle the brightest early-type galaxy at the southern end of the cluster, at separations of $\Delta \theta=4^{\prime \prime}$ and $\Delta \theta=6^{\prime \prime}$, respectively. The third is an arclet situated $\Delta \theta \sim 50^{\prime \prime}$ to the north of this features among a group of galaxies which are members of the cluster.

SDSS J1217+3641. An obvious arc-like feature was identified $\Delta \theta=33^{\prime \prime}$ from the cluster center; however, the gravitational lensing interpretation in this system is complicated by the presence of a foreground elliptical galaxy $\left(z_{\text {phot }}=0.12\right)$ at a separation of $\sim 10^{\prime \prime}$ from the candidate arc. Although the feature we identified is most likely an arc, it could also be a tidal tail or debris associated with the foreground object. Broad-band colors could be used to distinguish between these two possibilities, but an image to comparable depth in another filter (such as the $i$ band) is required.

Abell 1703. Abell 1703, a massive $X$-ray cluster with $L_{X}=$ $5.3 \times 10^{44} \mathrm{erg} \mathrm{s}^{-1}$ (Böhringer et al. 2000), is one of the most dramatic lensing clusters in our sample. A long giant arc at $\Delta \theta=35^{\prime \prime}$ stretches across the southeast end of the cluster. A second arc is identified at $\Delta \theta=13^{\prime \prime}$ from the BCG, with very high-surface-brightness knots at each end, which suggests a merging pair of images of the same background source. The large difference in angular separations of the two arcs is unlikely to be caused by very different source redshifts, especially considering that the two arcs have comparable surface brightnesses. Furthermore, the radius of curvature of the smaller separation arc and its merging pair morphology suggest that it is a minor axis tangential arc, rather than a radial arc or a lower redshift lensed galaxy (see, e.g., Dalal et al. 2004). If this interpretation is correct, the tangential critical curves in this lens will be highly flattened, with the wide arc demarcating the major axis, and the smaller arc indicating the minor axis. Such flattened cigar-shaped critical curves arise naturally from the shallow radial profiles and high ellipticity (Dalal \& Keeton 2003; Dalal et al. 2004) characteristic of clusters in $\Lambda$ CDM.

GHO 132029+315500. This cluster at $z=0.307$ was discovered in the photographic high-redshift cluster survey of Gunn et al. (1986), and has an X-ray luminosity $L_{X}=$ $4.4 \times 10^{44} \mathrm{erg} \mathrm{s}^{-1}$ (Böhringer et al. 2000). Even with the modest seeing of our WIYN image (seeing $=1$."00), we identify a 
definite giant arc at angular separation $\Delta \theta=21^{\prime \prime}$ to the east of the cluster center.

RX J1327.0+0211. This cluster at $z=0.260$ has an X-ray luminosity of $L_{\mathrm{X}}=7.7 \times 10^{44} \mathrm{erg} \mathrm{s}^{-1}$ (Popesso et al. 2004). An extremely high surface brightness arc is detected 4 " away from a cluster galaxy and curves slightly around it. This arc is at an angular separation of $\Delta \theta=93^{\prime \prime}$ to the northeast of the cluster center, which is clearly defined by a very luminous BCG, making it one of the largest separation giant arcs ever discovered, although the nearby galaxy is likely responsible for boosting the gravitational potential at such a large distance from the cluster center. The arc feature was bright enough to be detected in the SDSS photometry, and it has SDSS model magnitudes $(u, g, r, i, z)=(21.3,21.8,21.3,20.9,20.6)$.

NSC J134610+030555. A very faint arc is detected in our WIYN image (seeing $=0.66) \Delta \theta=19^{\prime \prime}$ from the center of this cluster at $z=0.232$.

Abell 1835. This cooling-flow cluster with $L_{X}=19.5 \times$ $10^{44} \mathrm{erg} \mathrm{s}^{-1}$ (Böhringer et al. 2000) is a well-known gravitational lens and has a prominent giant arc $31^{\prime \prime}$ from the cluster center, as well as several other candidate lensed features (Schmidt et al. 2001; Smith et al. 2005; Sand et al. 2005). The giant arc is clearly identified in our WIYN image in Figure 3 (seeing $=1.06^{\prime \prime}$ ), and it received the highest possible score of 6 .

Abell 1914. The arc candidates in this bright $X$-ray cluster $L_{\mathrm{X}}=8.9 \times 10^{44} \mathrm{erg} \mathrm{s}^{-1}$ (Böhringer et al. 2000) were first noted in the weak lensing study of Dahle et al. (2002). Sand et al. (2005) identify a faint tangential arc $\Delta \theta=28^{\prime \prime}$ to the southwest of the BCG, but no feature at this location was noted by either examiner of our WIYN image, which had a seeing of $0.62^{\prime \prime}$. However, we identified an arc oriented tangentially around a secondary concentration of galaxies which is located $\sim 1.5^{\prime}$ to the North and $\sim 1^{\prime}$ to the East of the cluster BCG. The arc is at separation $\Delta \theta=34^{\prime \prime}$ from the brightest galaxy in the secondary concentration. Sand et al. (2005) do not show the image of Abell 1914, but considering that the WFPC2 has an effective area of 134 " $\times 134$ " but with an "L"-shaped-field-of-view, it is conceivable that the arc which we detected in our WIYN image was not imaged by the WFPC2, explaining its absence from the Sand et al. (2005) compilation.

Abell 1942. The arc in this cluster was previously noted in the $V$-band imaging arc survey of survey of Smail et al. (1991).

SDSS J1446+3032. This cluster at $z_{\text {phot }}=0.47$ is one the most dramatic examples of strong gravitational lensing ever discovered. Five extended high surface brightness arcs are arranged about the center of the cluster in an ellipse with minor axis $\Delta \theta=13^{\prime \prime}$ and major axis $\Delta \theta=22^{\prime \prime}$. Additional $i$-band and $r$-band images of this cluster indicate that the arcs are very blue and that they all have similar colors, but it is not clear that whether they are images of the same source.

Abell 2070. A bright arc is detected $\Delta \theta=15^{\prime \prime}$ from the center of this cluster at $z=0.253$.

SDSS J1531+3414. This cluster at $z=0.335$ is another poster child gravitational lens, similar to SDSS J1446+3032, with a series of definite arcs arranged in a ellipse, with minor axis $\Delta \theta=11^{\prime \prime}$ and major axis $\Delta \theta=15^{\prime \prime}$. The arcs surround the very prominent $\mathrm{BCG}$, but there is a secondary grouping of galaxies $1^{\prime}$ to the southeast. These high surface brightness arcs are very wide and they are thus resolved, even in the poor seeing of our WIYN $g$-band image ( seeing $=1.2^{\prime \prime}$ ).

Abell 2141. Abell 2141 is an X-ray cluster with $L_{X}=$ $1.8 \times 10^{44} \mathrm{erg} \mathrm{s}^{-1}$ (Böhringer et al. 2000) at $z=0.159$. We identify an arc at $\Delta \theta=22^{\prime \prime}$ from the BCG. Four distinct blobs can be seen in the arc, which suggests a merging quad of multiple images of the same source.

SDSS J1557+2131. This cluster at $z=0.427$ has a complex morphology with a secondary mass concentration centered on a bright early-type galaxy $\Delta \theta=49^{\prime \prime}$ to the southwest. We identify three candidate lensing features in this cluster. The first is located $\Delta \theta=58^{\prime \prime}$ to the southwest of the BCG, close to the secondary mass concentration. The other two, located at $\sim 5^{\prime \prime}$ from a double-BCG, have extremely high surface brightness, and we anticipate that these could be highly magnified radial arcs. One of the images is more highly distorted than the other (the northernmost image) which is why it received a higher score (4 versus 2 ). It is especially intriguing that there is a double BCG: if the total mass distribution traces the orientation of these galaxies then the tangential critical curve would be elongated along the north-south direction, and the images which we identify would lie along the minor axiswhich is exactly where one expects radial arcs to be located (Dalal et al. 2004; Sand et al. 2005). Both of the candidate radial arcs were detected in the SDSS photometry, and they have $(u, g, r, i, z)$ magnitudes of $(22.0,21.9,21.9,21.8,22.6)$ and $(25.3,21.8,20.9,20.5,20.2)$, for the northernmost and southernmost images, respectively. The differences in color suggest that these are not the images of the same source, but distinct features. If either of these images are confirmed to be at high redshift, then they are highly magnified and will be useful for studies of high-redshift galaxies similar to the famous lensed image of the high redshift galaxy MS 1512-CB58 (Yee et al. 1996).

SDSSJ1602+4038. This cluster at $z=0.387$ has a faint arc $\Delta \theta=7^{\prime \prime}$ from the brightest cluster galaxy. There are hints of other suggestive features at the sky limit, making this a good target for deeper imaging.

Abell 2219. Abell 2219 is a luminous X-ray cluster at $z=0.234$ with $L_{\mathrm{X}}=14.3 \times 10^{44} \mathrm{erg} \mathrm{s}^{-1}$ (Böhringer et al. 2000). The two giant arcs in Abell 2219 were discovered by Smail et al. (1995). Smith et al. (2005) and Sand et al. (2005) summarize the locations and redshifts of other candidate arclets and multiply imaged features. In our WIYN image (seeing $=$ $0.69^{\prime \prime}$ ), we identified the giant arc at $z=2.73$ (Smith et al. 2005) as two features which received a score of 6 (northwest) and 5 (west), respectively (see Figure 3). The brighter arc at $z=1.070$ (Smith et al. 2005) to the southeast received a score of 5 .

RX J1652.2+4449. This X-ray cluster at $z=0.175$ with $L_{\mathrm{X}}=2.3 \times 10^{44} \mathrm{erg} \mathrm{s}^{-1}$ (Böhringer et al. 2000) has a bright arc $\Delta \theta=10^{\prime \prime}$ from its BCG. The arc is resolved in the $0.67^{\prime \prime}$ seeing of our WIYN image.

Abell 2261. Abell 2261 is a luminous X-ray cluster at $z=0.224$ with $L_{\mathrm{X}}=12.0 \times 10^{44} \mathrm{erg} \mathrm{s}^{-1}$ (Böhringer et al. 2000). The arc in Abell 2261 to the southwest of the BCG was first noted in the weak lensing study of Dahle et al. (2002). This arc is clearly identified in our WIYN image (seeing $=0.66^{\prime \prime}$ ) and it received a score of 6 . The Sand et al. (2005) summary identifies a second arc to the northeast with a much smaller length-to-width ratio $L / W=7.7$ (compared to $L / W=25.5$ for the southwesterly arc), but this feature was not identified in our WIYN image.

SDSSJ1747+5428. A small separation arc $\Delta \theta=5^{\prime \prime}$ east of the BCG is detected in this cluster with photometric redshift $z_{\text {phot }}=0.31$.

SDSSJ2111-01115. This cluster at $z_{\text {phot }}=0.68$ was targeted for imaging at WIYN after it was identified as an arc candidate 


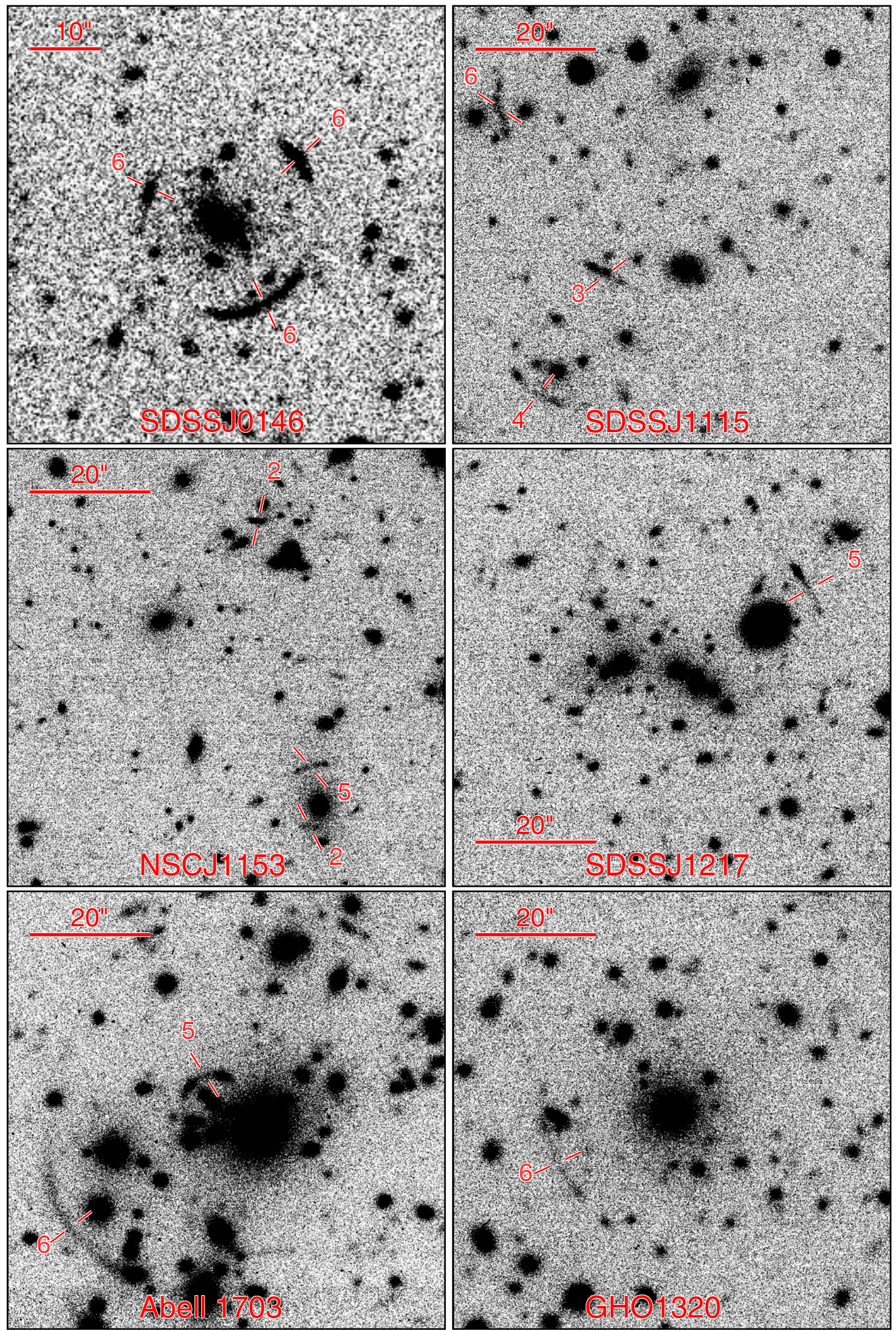

Figure 3. Cutout images of the definite lensing clusters listed in Table 1. Strong lensing features are indicated by lines and a number which is the score of the feature. North is up and east is to the left. The scalebar indicates the size of the cutouts and varies from $10^{\prime \prime}$ to $30^{\prime \prime}$, depending on the angular separation of the arcs. The name of the cluster is indicated at the bottom of each cutout.

(A color version of this figure is available in the online journal) 


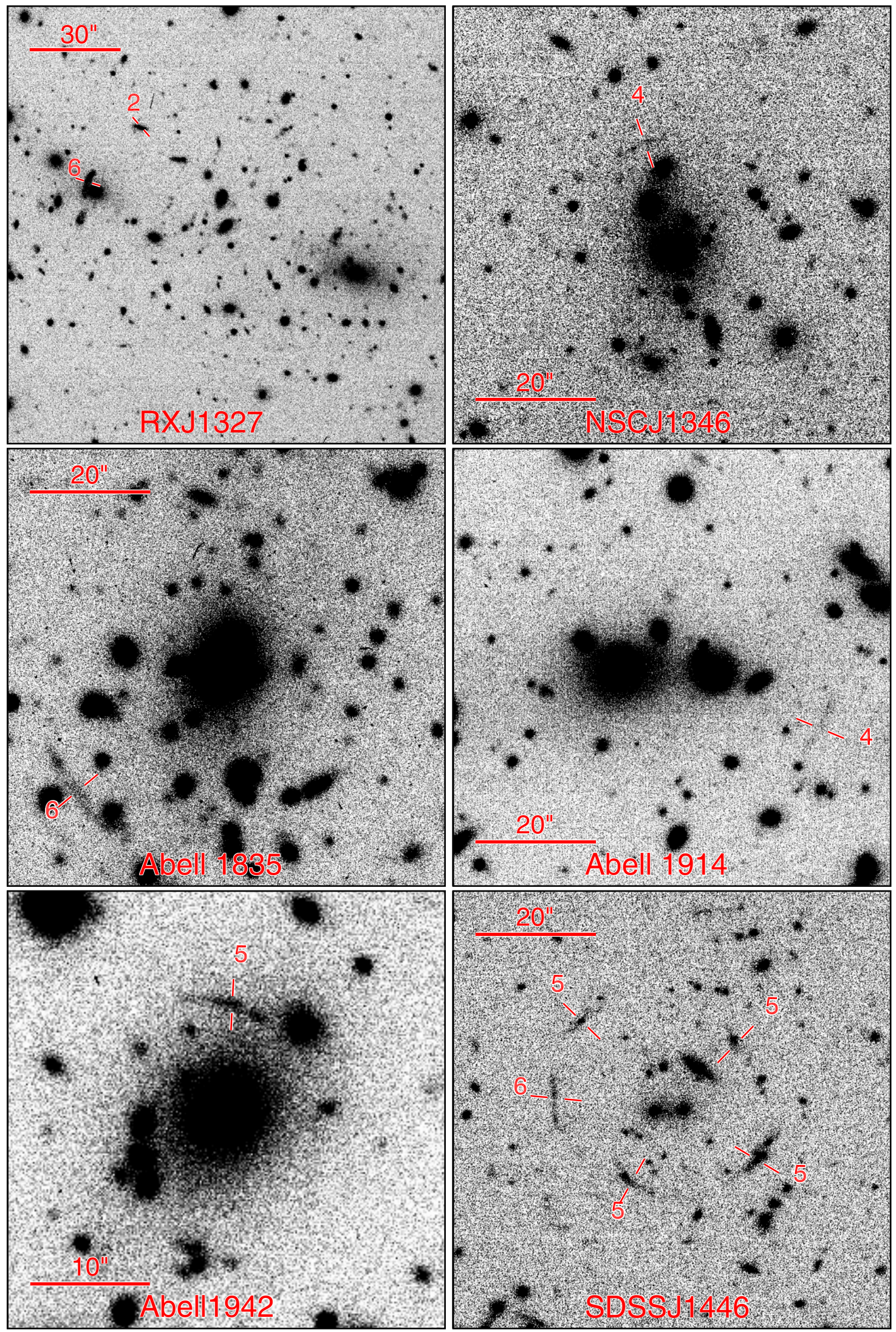

Figure 3. (Continued) 

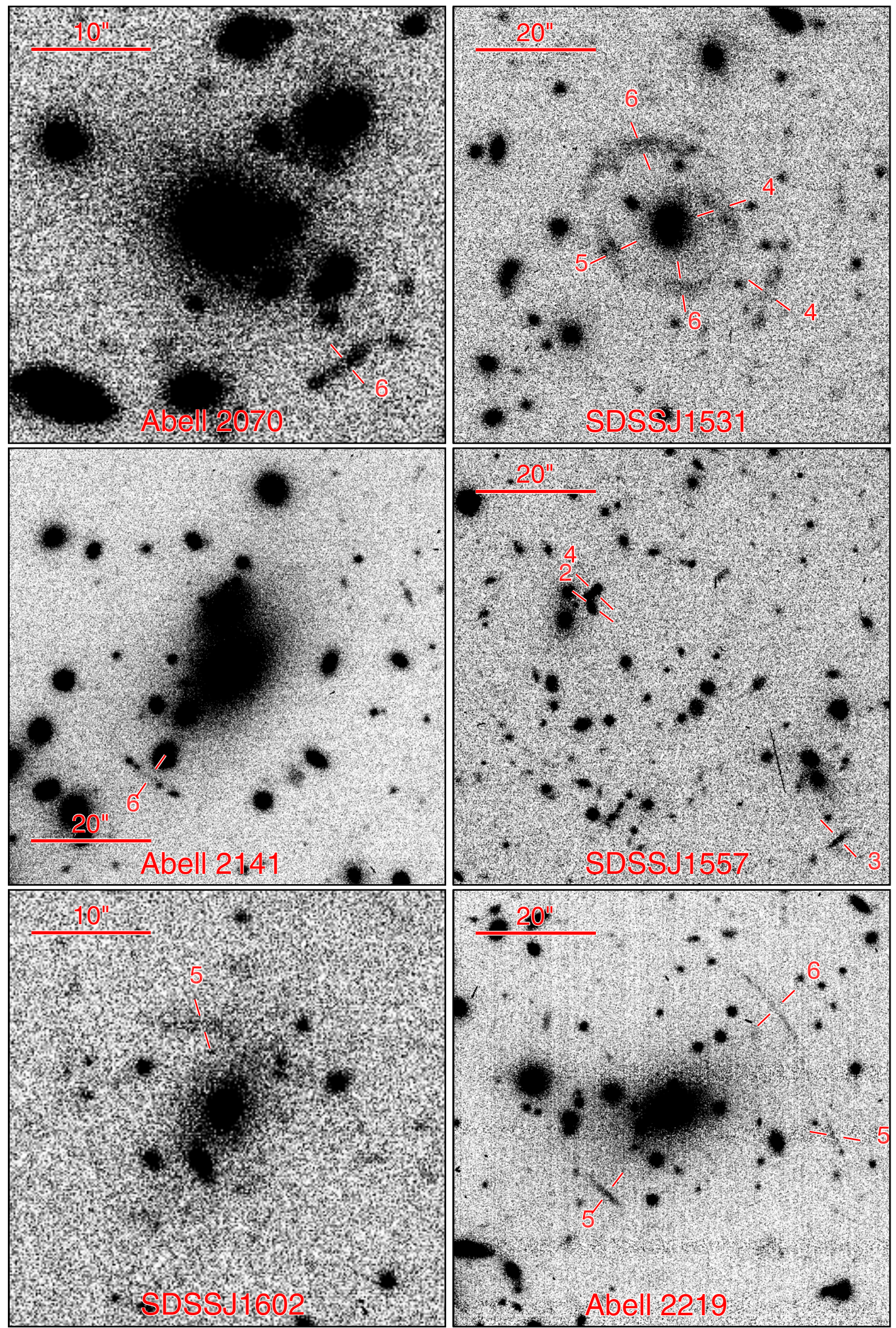

Figure 3. (Continued) 

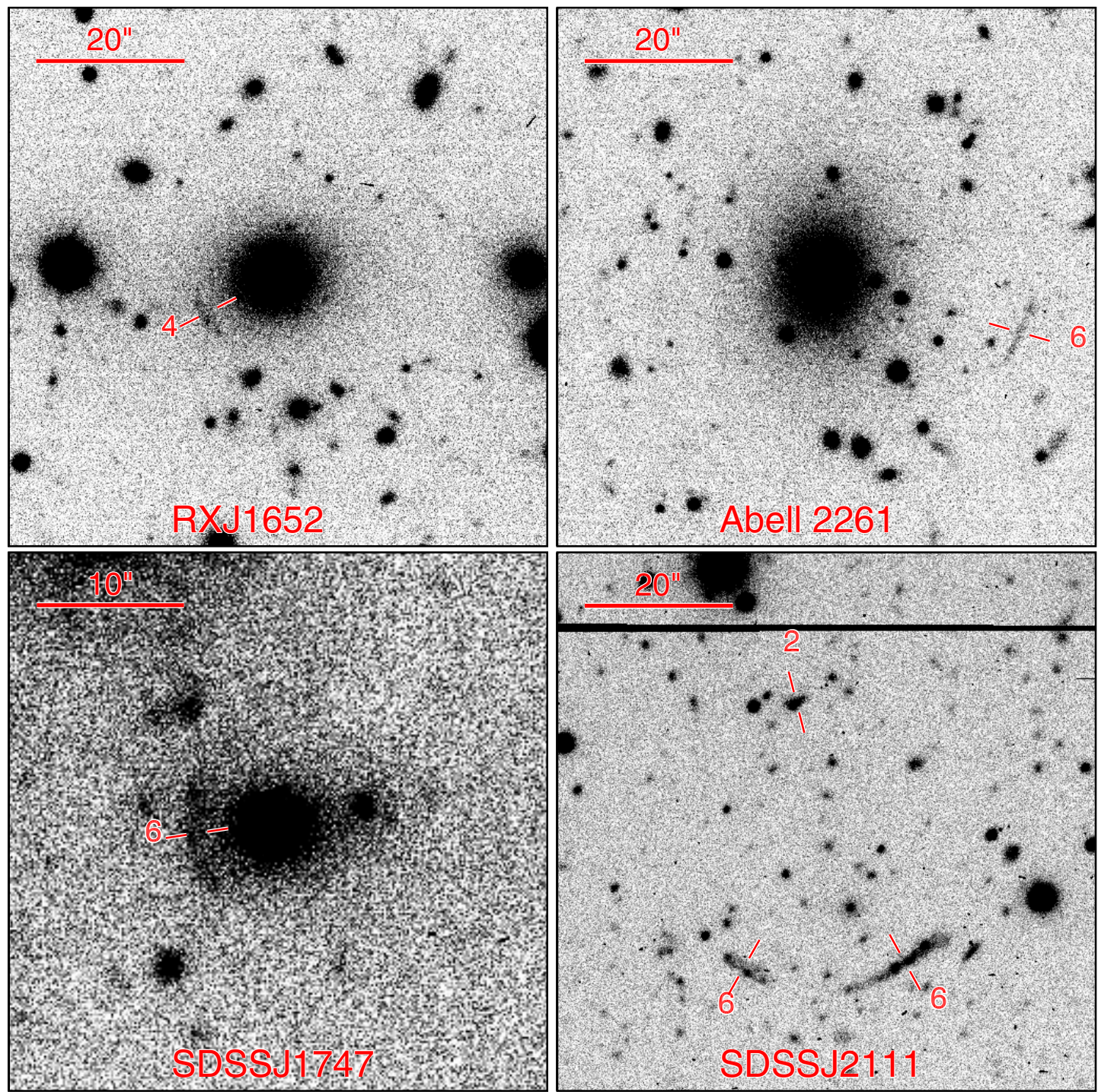

Figure 3. (Continued)

in the visual inspection search of the SDSS coadded southern strip imaging (Estrada et al. 2007). Our WIYN image shows two very high-surface-brightness arcs south of the BCG with angular separations of $\Delta \theta=11^{\prime \prime}$ and $\Delta \theta=16^{\prime \prime}$, for the longer and shorter arc, respectively. Additional images in $r$ band and $i$ band were taken, and both arcs are very blue and have similar colors, suggesting that they are images of the same source. Furthermore, three bright high-surface-brightness knots can be identified in both arcs. Based on the additional imaging, we identify a counter-image candidate, which is a high-surfacebrightness blue feature $\Delta \theta=28^{\prime \prime}$ north of the BCG. If all three of these features are images of the same source, they are not very well centered on the BCG, which could indicate that the BCG is offset significantly from the center of the dark matter potential well.

\subsection{Likely Lenses}

SDSS J1040+3405. A short arc $\Delta \theta=26^{\prime \prime}$ from the BCG is identified in our UH88 $V$-band image (seeing $=1.10^{\prime \prime}$ ) of this cluster at $z=0.445$, which is one of the richest members of our cluster catalog with $z>0.4$.
SDSS J1131+3554. This cluster at $z_{\text {phot }}=0.49$ shows a likely giant arc $\Delta \theta=12^{\prime \prime}$ from the double-BCG which defines the cluster center. However, the interpretation is complicated by the superposition with a bright star and the relatively poor seeing of our WIYN image $\left(1.10^{\prime \prime}\right)$.

Abell 1351. This bright $X$-ray cluster at $z=0.322$ has $L_{\mathrm{X}}=5.2 \times 10^{44} \mathrm{erg} \mathrm{s}^{-1}$ (Böhringer et al. 2000). In our UH88 $V$-band image (seeing $=0.97^{\prime \prime}$ ) of this cluster, we identify an arc-like feature $29^{\prime \prime}$ to the southwest of the cluster center, which received a score of 3 . The weak gravitational lensing study of Dahle et al. (2002) noted a "bright red gravitational arc offset from the cluster light center." Comparing our UH88 $V$-band image of this cluster to a color composite image kindly provided by Håkon Dahle, we see the arc-like feature we identified and we marginally detect this additional red arc noted by Dahle et al. (2002). This red arc is outside the field of view of our image in Figure 3, and is oriented around an elliptical galaxy further to the north. Since it is near the detection limit of our image, it was not noted by either examiner in our search.

SDSS J1150+0650. The arclet identified south of the BCG in this cluster at $z=0.301$ is notable for its large separation $\Delta \theta=25^{\prime \prime}$. 




Figure 4. Same as Figure 3, but for the likely lensing clusters listed in Table 2. (A color version of this figure is available in the online journal) 


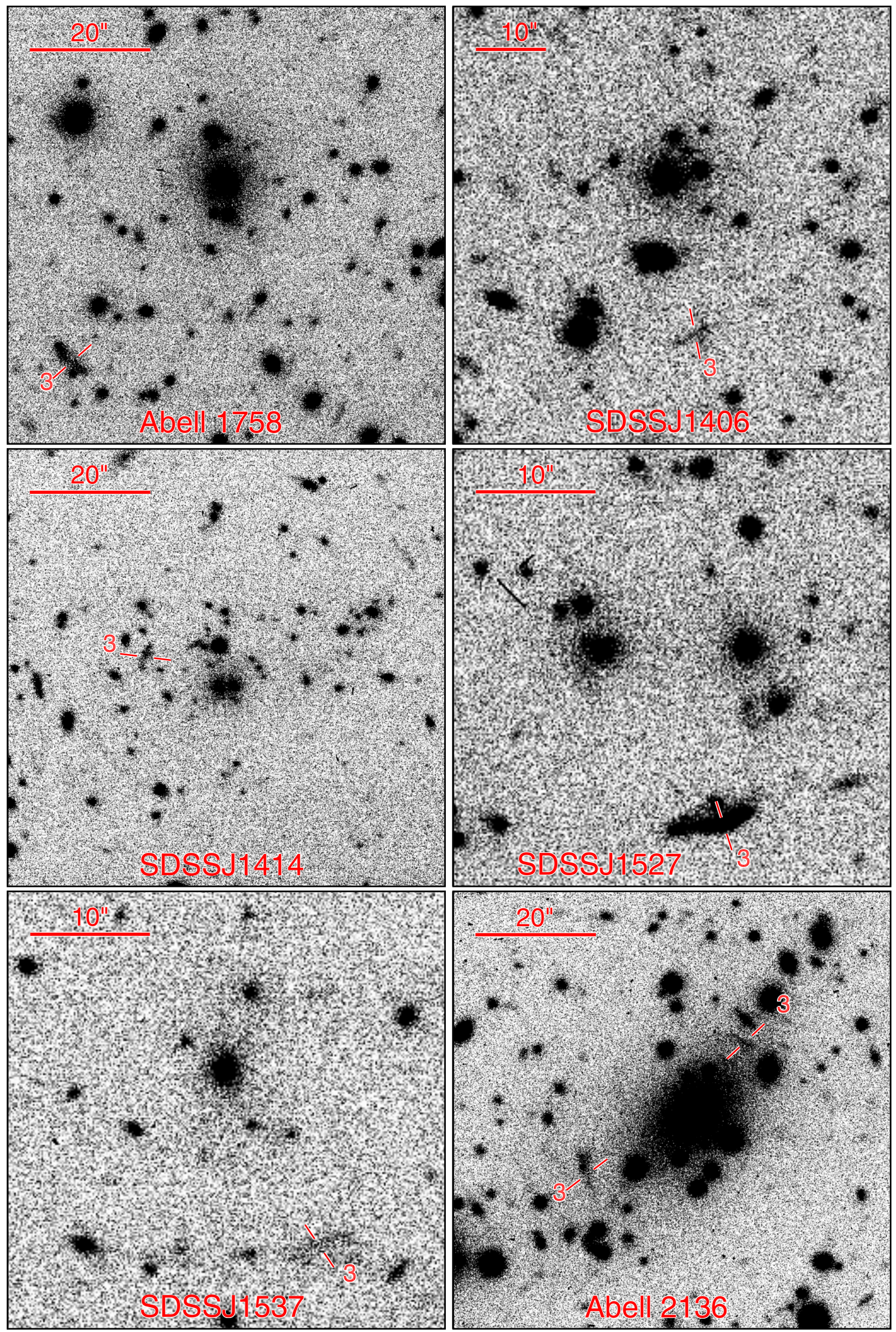

Figure 4. (Continued) 

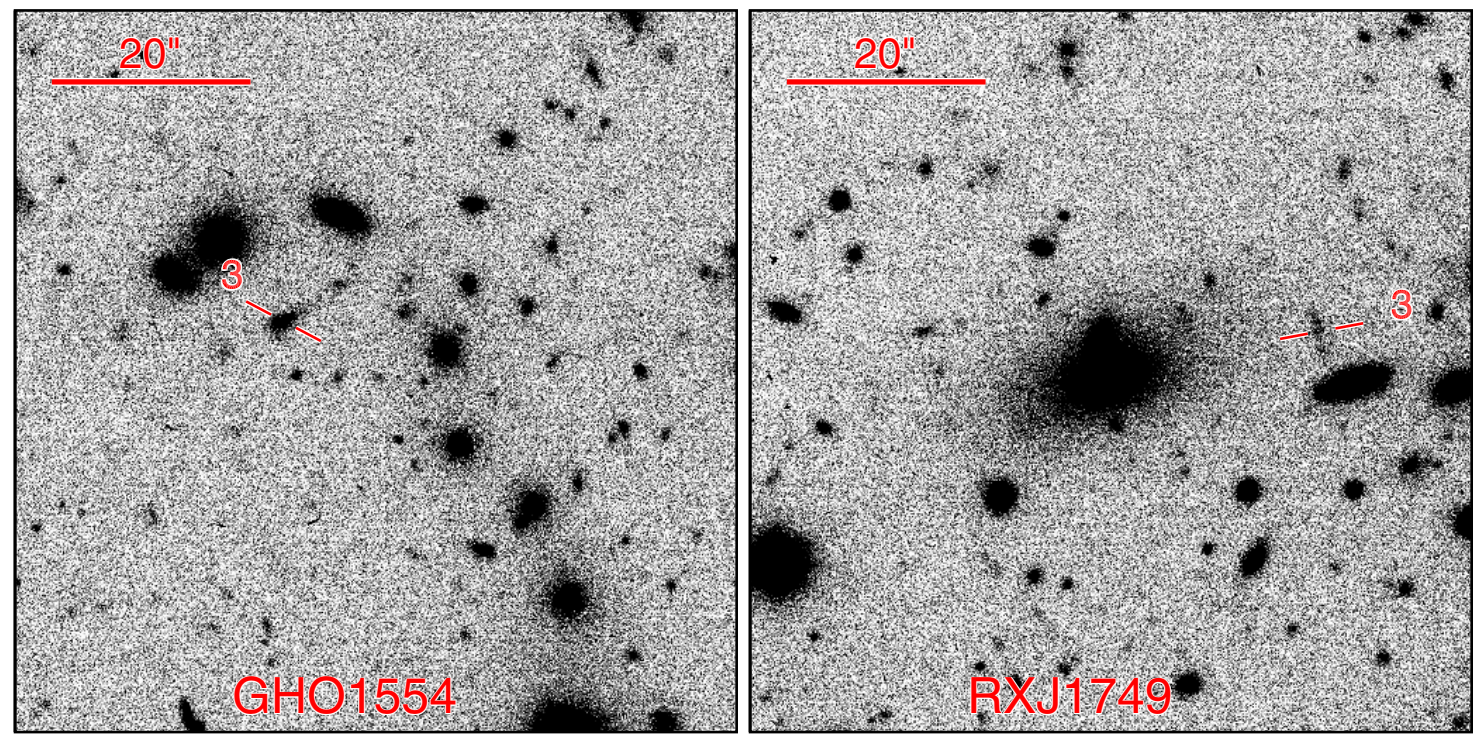

Figure 4. (Continued)

Abell 1550. Despite the poor seeing (1.30") of our WIYN image of Abell 1550, we identify a faint arc $\Delta \theta=16^{\prime \prime}$ northeast of the BCG. This cluster is at $z=0.262$ and has $L_{\mathrm{X}}=3.4 \times 10^{44} \mathrm{erg} \mathrm{s}^{-1}$ (Böhringer et al. 2000).

SDSS J1258+4702. The WIYN image of this cluster at $z_{\text {phot }}=0.32$ has the worst seeing $\left(1.39^{\prime \prime}\right)$ of any the candidate cluster lenses we identified, but we still identified a small separation arc $\Delta \theta=3^{\prime \prime}$ from the BCG.

Abell 1758. In our WIYN image (seeing $=0.85^{\prime \prime}$ ), Abell 1758 appears as a double cluster which is possibly in the process of merging. Both subclumps are concentrated around a bright early type galaxy, and the total X-ray luminosity is $L_{\mathrm{X}}=11.2 \times 10^{44} \mathrm{erg} \mathrm{s}^{-1}$ (Böhringer et al. 2000). Dahle et al. (2002) noted the presence of a blue arc associated with the northwest mass clump, which received a score of 3 in our arc search.

SDSS J1406+3945. A short arc $\Delta \theta=22^{\prime \prime}$ south of the BCG is identified in our UH88 $V$-band image of this cluster at $z=0.427$

SDSS J1414+2703. This cluster at $z_{\text {phot }}=0.46$ shows a likely arc $\Delta \theta=14^{\prime \prime}$ northeast of the double-BCG, which defines the cluster center. There are many other features at or near the sky limit which are highly suggestive of strong lensing, making this an excellent candidate for deeper multicolor imaging.

SDSS J1527+0652. This cluster at $z_{\text {phot }}=0.40$ is one of the richest clusters in our catalog. An extremely high-surfacebrightness blue arc candidate is identified $\Delta \theta=17^{\prime \prime}$ south of the BCG. This arc candidate was detected in the SDSS imaging and has magnitudes $(u, g, r, i, z)=(24.8,20.9,20.7,20.9,21.1)$, making it an easy target for spectroscopic follow-up. This arc was classified as likely rather than definite: its high surface brightness led us to wonder whether it might be a foreground edge-on spiral galaxy.

SDSS J1537+3926. We detect a faint arc which is optically resolved and very near the sky limit of our WIYN image in this cluster at $z=0.444$. The arc is located $\Delta \theta=17^{\prime \prime}$ from a bright cluster galaxy located in a secondary concentration of galaxies $1.5^{\prime}$ north of the cluster center.

Abell 2136. Abell 2136 at $z=0.229$ has an X-ray luminosity of $L_{X}=1.7 \times 10^{44} \mathrm{erg} \mathrm{s}^{-1}$ (Böhringer et al. 2000). Two very likely arcs are identified to the northwest and southeast, at separations of $\Delta \theta=14^{\prime \prime}$ and $\Delta \theta=21^{\prime \prime}$, respectively.

GHO 155414+40530. An ambiguous arc-like feature overlaps a cluster galaxy at $\Delta \theta=40^{\prime \prime}$ northeast of the BCG in this cluster at $z=0.393$.

RXC J1749.3+4245. This cluster at $z=0.230$ has an X-ray luminosity $L_{\mathrm{X}}=1.9 \times 10^{44} \mathrm{erg} \mathrm{s}^{-1}$ (Böhringer et al. 2000). A short arc is identified $\Delta \theta=22^{\prime \prime}$ west of the cluster center.

\subsection{Possible Lenses}

SDSS J1008+4529. This pair of arcs was missed by one of the examiners because the lensing features are centered on a galaxy which is several arcminutes from the galaxy identified as the BCG by our cluster finding algorithm. However, this $z=0.479$ system has a complex morphology and hence the location of the cluster center is somewhat ambiguous. Two arc-like features are identified at separations $\Delta \theta=8^{\prime \prime}$ (eastern) and $\Delta \theta=10^{\prime \prime}$ (northwest) of the cluster galaxy.

SDSS J1240+4250. This cluster at $z=0.406$ is $2.9^{\prime}$ southwest of the known galaxy cluster NSC J124039+425228 with published redshift $z=0.3955$ (Gal et al. 2003). The similarity of the redshifts suggests a centering error in the NSC cluster catalog. This cluster has several very suggestive high surface brightness arc candidates. The orientation/geometry of these features does not obviously suggest a lensing interpretation, although the cluster has a complex morphology.

SDSSJ1513+0525. A very high-surface-brightness arc candidate is identified $\Delta \theta=38^{\prime \prime}$ to the north of the BCG of this cluster at $z_{\text {phot }}=0.49$. Because of the large angular separation and the fact that the feature is so bright $(u, g, r, i, z)=$ $(22.8,21.5,20.2,19.7,19.4)$, we were not certain of the lensing interpretation and classified this system as a possible lens.

\subsection{Known Lenses Which Were Missed}

Abell 773. Smith et al. (2005) identified several arcs and many candidate multiple image systems in HST Wide Field Planetary Camera 2 (WFPC2) imaging (F702W filter) of this bright X-ray cluster $\left(L_{\mathrm{X}}=7.0 \times 10^{44} \mathrm{erg} \mathrm{s}^{-1}\right)$. Our WIYN image of this cluster has a seeing of $0.79^{\prime \prime}$ and the counterparts to the majority of the lensed features from Smith et al. can be 

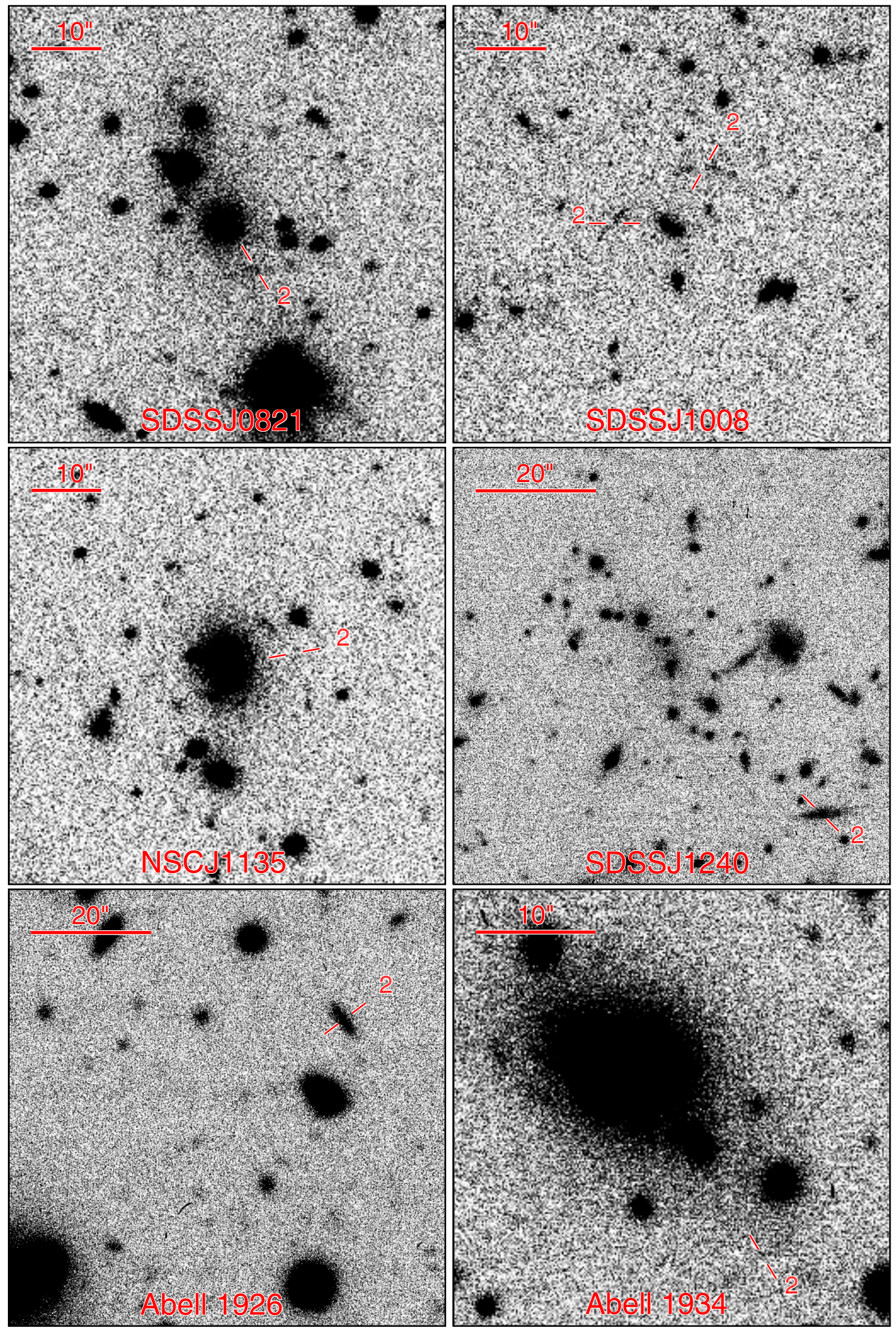

Figure 5. Same as Figure 3, but for the possible lensing clusters listed in Table 3.

(A color version of this figure is available in the online journal) 

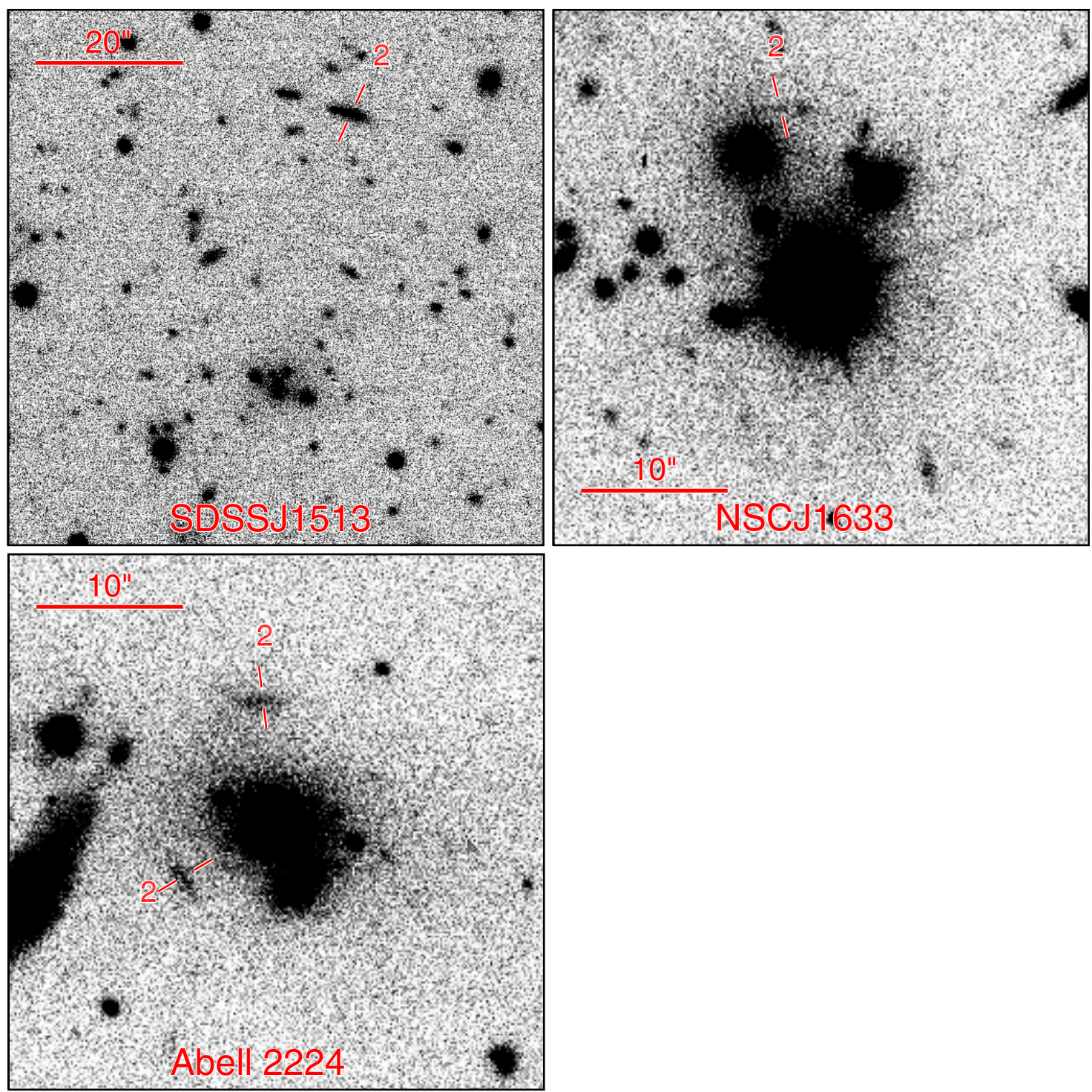

Figure 5. (Continued)

identified when comparing the HST and WIYN images side by side. But only those features with the largest length-to-width ratios could be convincingly identified as arcs or arclets given our image quality. Five features were given a score of 1 when inspected by one examiner, whereas the second examiner found only the most conspicuous of these and gave it a score of 1 . Thus the highest score of any feature was 2 , with a score of 1 from each examiner, which is not sufficient to make it into our lens samples.

Z3146. Sand et al. (2005) identified a single faint (integrated magnitude $\mathrm{F} 606=23.55$ ) tangential arc at $\Delta \theta=26^{\prime \prime}$ to the southeast of the center of this well known cooling flow cluster. An arclet-like feature can be seen at roughly this location in our WIYN image (seeing $=1.09^{\prime \prime}$ ); however, it is near the sky limit and does not appear very tangentially distorted. Neither of the image examiners identified this feature.

Abell 1763. Smith et al. (2005) list several candidate multipleimage systems in this cluster and Sand et al. (2005) identified one of them as a tangential arc with length-to-width ratio $L / W=8.7$ and magnitude F702 $=24.87$. However, this feature is extremely faint and the lensing interpretation is not very compelling, even in the HST image. No candidate lensing features were identified in our WIYN image $\left(\right.$ seeing $\left.=0.64^{\prime \prime}\right)$ of this cluster.

\section{SUMMARY AND CONCLUSIONS}

We have conducted a systematic deep imaging survey for lensed arcs and arclets in a large optically selected sample of distant clusters. A total of 240 clusters were imaged (195 from WIYN and 45 from UH88), of which 141 had sub-arcsecond image quality. Our survey uncovered 22 definite lensing clusters (6 previously known), 14 likely lenses ( 2 previously known), and 9 possible lensing clusters. It is probable that $\gtrsim 50 \%$ of our likely and possible samples are indeed cluster lenses, so that the number of new cluster lenses discovered here is $\sim 30$. This is a substantial contribution to the total number of lensing clusters known, which was previously $\sim 50$. Among these new systems are some of the most dramatic examples of gravitational lensing ever discovered. Clusters such as SDSS J1115+5319, Abell 1703, SDSS J1446+3032, and SDSS J1531+3414, which 
have multiple bright arcs at large angular separation, will likely become "poster-child" gravitational lenses similar to Abell 1689 and CL0024+1654.

At first glance it may seem surprising that comparably few of the lensing clusters presented here are X-ray clusters. In particular, for our 22 definite lensing clusters only 9 are detected in X-rays, all of which were identified in the NORAS catalog (Böhringer et al. 2000), which comprises the largest overlapping area with the SDSS imaging. This relative paucity of X-ray detections arises because our optical cluster catalog extends to significantly higher redshift $(z \lesssim 0.6)$ than the Xray cluster samples we matched to $(z \lesssim 0.3)$. Specifically, all of the lenses which are detected as X-ray clusters have $z \leqslant 0.307$, whereas 10 out of our 22 definite lenses have $z>0.307$. The average X-ray luminosity for the detected lenses is $L_{\mathrm{X}}=8.5 \times 10^{44} \mathrm{erg} \mathrm{s}^{-1}$. Böhringer et al. (2000) estimated the completeness of the NORAS sample to be about $50 \%$ at an X-ray flux of $F_{\mathrm{X}}(0.1-2.4 \mathrm{keV})=3 \times 10^{-12} \mathrm{erg} \mathrm{s}^{-1} \mathrm{~cm}^{-2}$. This flux limit corresponds to $L_{\mathrm{X}}>8.2 \times 10^{44} \mathrm{erg} \mathrm{s}^{-1}$ for redshifts $z>0.30$. It is thus not surprising that the half of our lensing cluster sample with $z>0.30$ are not detected as X-ray clusters, since only extremely luminous X-ray clusters would be detected by NORAS. However, at lower redshift $z \leqslant 0.307$ where NORAS can detect more typical X-ray luminosities, we find that 9 out of 12 cluster lenses are indeed X-ray clusters.

There are several advantages to our ground-based survey strategy. First and foremost, the cosmological volume accessible to our survey is $\sim 2 h^{-1} \mathrm{Gpc}^{3}\left(0.1 \lesssim z \lesssim 0.6 ; \sim 8000 \mathrm{deg}^{2}\right)$, which is more than an order of magnitude larger than any previous search. This enables us to characterize the lensing cross-sections of a more generic population of galaxy clusters, whereas most previous arc searches have been dominated by nearby bright X-ray clusters (i.e. Le Fevre et al. 1994; Luppino et al. 1999; Sand et al. 2005). Second, inspection of Figures 3-5 reveals that our survey primarily discovers highsurface-brightness arcs which are resolved in ground-based image quality. Indeed, several of the arcs published here have $g \lesssim 21$, making them among the brightest giant arcs ever discovered. Spectroscopic observations of the bright arcs in our sample, which are required both to confirm the lensing hypothesis and to construct accurate mass models, will be much easier than for arcs discovered in HST images, which tend to be $\gtrsim 2$ magnitudes fainter.

But considering that we are imaging from the ground with a median seeing of $0.90^{\prime \prime}$, our completeness for identifying lensed images will surely be lower than a survey from space, such as the WFPC2 archive search of Sand et al. (2005). However, 8 out of the 11 previously known lenses which were imaged in our survey were recovered, and most of the arcs/arclets which we missed had magnitudes close to or below our surface brightness limit. To quantify the selection function of our imaging survey we intend to add synthetic lensed images to our data and conduct a similar visual inspection search on this mock data set, which will be presented in a future work (Gladders et al. 2008b, in preparation).

Although the input cluster catalog to our imaging survey is richness selected, our survey strategy is to image a large enough sample of clusters, thus allowing us to drive down the limiting survey mass far enough that the scatter between richness and mass does not bias our sample. While this ensures that all clusters massive enough to produce giant arcs are imaged, lenses will nevertheless be missed because we image in a single filter in ground-based seeing. However, to a good approximation the properties of the lensed sources are decoupled from the properties of the lens. Thus rather than being mass-limited or flux-limited our lens sample will be strong lensing cross-section limited. It is straightforward to use ray tracing simulations to model the statistical properties of a lensing selected sample of clusters (e.g., Hennawi et al. 2007a, 2007b). Most importantly, the selection function of our survey will be determined by the properties of the dark matter distribution rather than on the relationship between mass and cluster observable (i.e. light).

The cosmological applications of this sample of $\sim 30$ new lensing clusters are many. The sample size and redshift coverage $(z \lesssim 0.6$; see Figure 2 ) will allow us to characterize the strong lensing properties of a statistical sample of low-redshift clusters, which is important in light of the recent suggestion by Gladders et al. (2003) that most cluster strong lensing occurs at high redshift. Our low-redshift lensing survey is thus a necessary complement to ongoing high-redshift giant arc searches such as the RCS and RCS2 (Gladders \& Yee 2000; Gladders et al. 2003; Gladders \& Yee 2005), as well as the Massive Cluster Survey (MACS) (Ebeling et al. 2001). The abundance and statistics of giant arcs will be addressed in a future paper (Gladders et al. 2008b, in preparation). HST imaging of the most dramatic lenses in our sample will likely uncover many more arcs and candidate multiple images. Using these image positions, detailed models can measure the distribution of dark matter in each cluster (e.g. Tyson et al. 1998; Smith et al. 2001; Sand et al. 2004; Broadhurst et al. 2005a) and around the cluster galaxies (Natarajan et al. 2004), and stronger constraints can be obtained if the strongly lensed image positions are combined with larger-scale weak-lensing measurements (Kneib et al. 2003; Gavazzi et al. 2003; Smith et al. 2005; Broadhurst et al. 2005b; Natarajan et al. 2006; Dalal et al. 2008, in preparation). This will provide much improved statistics on the properties of the dark matter distribution in clusters such as ellipticity, radial profile, concentrations, and substructure, enabling statistical comparisons to theoretical predictions (Hennawi et al. 2007b; Natarajan et al. 2006). These comparisons are of particular interest considering that the large $\left(c_{\mathrm{NFW}}>14\right)$ concentrations recently measured in cluster lenses (Gavazzi et al. 2003; Kneib et al. 2003; Broadhurst et al. 2005a) are significantly higher than the expectations from CDM (Oguri et al. 2005; Hennawi et al. 2007b; Dalal et al. 2008, in preparation).

Our survey for clusters lenses is only half complete, and we expect to find a comparable number of lenses to the $\sim 30$ published here upon its completion. By conducting the largest giant arc search to date, we will help transform strong lensing by galaxy clusters from the study of a handful of rare systems, into a powerful statistical probe of the formation of structure in the universe.

We are grateful to Nikhil Padmanabhan, Erin Sheldon, and David Schlegel for help with photometric redshifts for SDSS galaxies. We would like to thank Håkon Dahle for kindly providing a color image of Abell 1351. Thanks also to Steve Howell, George Will, Doug Williams, and the other members of the WIYN observatory staff for assistance and superb technical support over the course of our survey runs. J.F.H. and M.G. are supported by NASA through Hubble Fellowship grants \# HF-01172.01-A and HF-01184.01, respectively, awarded by the Space Telescope Science Institute, which is operated by the Association of Universities for Research in Astronomy, Inc., for NASA, under contract NAS 5-26555. D.P.S. acknowledges support from the National Science Foundation grant AST- 
0607634. I.K. was in part financially supported by the 21 st Century COE Program of Nagoya. This work was supported in part by the Department of Energy contract DE-AC0276SF00515. Use of the UH $2.2 \mathrm{~m}$ telescope for the observations is supported by NAOJ.

Based in part on observations obtained at the $3.5 \mathrm{~m}$ and $0.9 \mathrm{~m}$ WIYN Telescopes. The WIYN Observatory is a joint facility of the University of Wisconsin-Madison, Indiana University, Yale University, and the National Optical Astronomy Observatories (NOAO).

The authors wish to recognize and acknowledge the very significant cultural role and reverence that the summit of Mauna Kea has always had within the indigenous Hawaiian community. We are most fortunate to have the opportunity to conduct observations from this mountain.

Funding for the SDSS and SDSS-II has been provided by the Alfred P. Sloan Foundation, the Participating Institutions, the National Science Foundation, the U.S. Department of Energy, the National Aeronautics and Space Administration, the Japanese Monbukagakusho, the Max Planck Society, and the Higher Education Funding Council for England. The SDSS Web Site is http://www.sdss.org/.

The SDSS is managed by the Astrophysical Research Consortium for the Participating Institutions. The Participating Institutions are the American Museum of Natural History, Astrophysical Institute Potsdam, University of Basel, Cambridge University, Case Western Reserve University, University of Chicago, Drexel University, Fermilab, the Institute for Advanced Study, the Japan Participation Group, Johns Hopkins University, the Joint Institute for Nuclear Astrophysics, the Kavli Institute for Particle Astrophysics and Cosmology, the Korean Scientist Group, the Chinese Academy of Sciences (LAMOST), Los Alamos National Laboratory, the Max Planck Institute for Astronomy (MPIA), the Max Planck Institute for Astrophysics (MPA), New Mexico State University, Ohio State University, University of Pittsburgh, University of Portsmouth, Princeton University, the United States Naval Observatory, and the University of Washington.

\section{REFERENCES}

Abazajian, K., et al. 2003, AJ, 126, 2081

Abazajian, K., et al. 2004, AJ, 128, 502

Abazajian, K., et al. 2005, AJ, 129, 1755

Adelman-McCarthy, J. K., et al. 2006, ApJS, 162, 38

Adelman-McCarthy, J. K., et al. 2008, in preparation

Abell, G. O., Corwin Jr., H. G., \& Olowin, R. P. 1989, ApJS, 70, 1

Bartelmann, M., Huss, A., Colberg, J. M., Jenkins, A., \& Pearce, F. R. 1998 A\&A, 330, 1

Blain, A. W., Kneib, J.-P., Ivison, R. J., \& Smail, I. 1999, ApJ, 512, L87

Broadhurst, T., et al. 2005a, ApJ, 621, 53

Broadhurst, T., Takada, M., Umetsu, K., Kong, X., Arimoto, N., Chiba, M., \& Futamase, T. 2005b, ApJ, 619, L143

Böhringer, H., et al. 2000, ApJS, 129, 435

Böhringer, H., et al. 2004, A\&A, 425, 367

Castander, F. J., et al. 2001, AJ, 121, 2331

Crawford, C. S., Edge, A. C., Fabian, A. C., Allen, S. W., Bohringer, H., Ebeling, H., McMahon, R. G., \& Voges, W. 1995, MNRAS, 274, 75

Dahle, H., Kaiser, N., Irgens, R. J., Lilje, P. B., \& Maddox, S. J. 2002, ApJS, 139,313

Dalal, N., \& Keeton, C. R. 2003, arXiv astro-ph/0312072

Dalal, N., Holder, G., \& Hennawi, J. F. 2004, ApJ, 609, 5

Dalal, N., Hennawi, J. F., Cole, S., \& Bode, P. 2008, in preparation

Ebeling, H., Edge, A. C., \& Henry, J. P. 2001, ApJ, 553, 668

Eisenstein, D. J., et al. 2001, AJ, 122, 2267

Estrada, J., et al. 2007, ApJ, 660, 1176

Fukugita, M., Ichikawa, T., Gunn, J. E., Doi, M., Shimasaku, K., \& Schneider, D. P. 1996, AJ, 111, 1748
Gal, R. R., de Carvalho, R. R., Lopes, P. A. A., Djorgovski, S. G., Brunner, R. J., Mahabal, A., \& Odewahn, S. C. 2003, AJ, 125, 2064

Gavazzi, R., Fort, B., Mellier, Y., Pelló, R., \& Dantel-Fort, M. 2003, A\&A, 403, 11

Gladders, M. D., \& Yee, H. K. C. 2000, AJ, 120, 2148

Gladders, M. D., Hoekstra, H., Yee, H. K. C., Hall, P. B., \& Barrientos, L. F. 2003, ApJ, 593, 48

Gladders, M. D., Yee, H. K. C., Mahjumdar, S., Barrientos, L. F., Hoekstra, H., Hall, P. B., \& Infante, L. 2007, ApJ, 655, 128

Gladders, M. D., \& Yee, H. K. C. 2005, ApJS, 157, 1

Gladders, M. D. et al. 2008a, in preparation

Gladders, M. D. et al. 2008b, in preparation

Gunn, J. E., Hoessel, J. G., \& Oke, J. B. 1986, ApJ, 306, 30

Gunn, J. E., et al. 1998, AJ, 116, 3040

Gunn, J. E., et al. 2006, AJ, 131, 2332

Hennawi, J. F., Dalal, N., \& Bode, P. 2007a, ApJ, 654, 93

Hennawi, J. F., Dalal, N., Bode, P., \& Ostriker, J. P. 2007b, ApJ, 654, 714

Horesh, A., Ofek, E. O., Maoz, D., Bartelmann, M., Meneghetti, M., \& Rix, H.-W. 2005, ApJ, 633, 768

Ho, S., \& White, M. 2005, Astroparticle Physics, 24, 257

Hogg, D. W., Finkbeiner, D. P., Schlegel, D. J., \& Gunn, J. E. 2001, AJ, 122,2129

Howell, S. B., Everett, M. E., Tonry, J. L., Pickles, A., \& Dain, C. 2003, PASP, 115,1340

Ivezić, Ž., et al. 2004, Astron. Nachr., 325, 583

Kneib, J., et al. 2003, ApJ, 598, 804

Le Fevre, O., Hammer, F., Angonin, M. C., Gioia, I. M., \& Luppino, G. A 1994, ApJ, 422, L5

Li, G.-L., Mao, S., Jing, Y. P., Bartelmann, M., Kang, X., \& Meneghetti, M. 2005, ApJ, 635, 795

Li, G. L., Mao, S., Jing, Y. P., Mo, H. J., Gao, L., \& Lin, W. P. 2006, MNRAS, 372, L73

Luppino, G. A., Gioia, I. M., Hammer, F., Le Fèvre, O., \& Annis, J. A 1999, A\&AS, 136, 117

Lupton, R. H., Gunn, J. E., Ivezić, Z., Knapp, G. R., Kent, S., \& Yasuda, N. 2001 ASP Conf. Ser. 238: Astronomical Data Analysis Software and Systems X, 10,269

Lynds, R., \& Petrosian, V. 1989, ApJ, 336, 1

Metcalfe, L., et al. 2003, A\&A, 407, 791

Natarajan, P., Kneib, J.-P., Smail, I., \& Ellis, R. 2004, arXiv astro-ph/ 0411426

Natarajan, P., De Lucia, G., \& Springel, V. 2006, arXiv astro-ph/0604414

Oguri, M., Lee, J., \& Suto, Y. 2003, ApJ, 599, 7

Oguri, M., Takada, M., Umetsu, K., \& Broadhurst, T. 2005, ApJ, 632, 841

Padmanabhan, N., et al. 2005, MNRAS, 359, 237

Pier, J. R., Munn, J. A., Hindsley, R. B., Hennessy, G. S., Kent, S. M., Lupton, R. H., \& Ivezić, Ž. 2003, AJ, 125, 1559

Popesso, P., Böhringer, H., Brinkmann, J., Voges, W., \& York, D. G. 2004, A\&A, 423, 449

Saha, A., Armandroff, T., Sawyer, D. G., \& Corson, C. 2000, Proc. SPIE, 4008, 447

Sand, D. J., Treu, T., Smith, G. P., \& Ellis, R. S. 2004, ApJ, 604, 88

Sand, D. J., Treu, T., Ellis, R. S., \& Smith, G. P. 2005, ApJ, 627, 32

Santos, M. R., Ellis, R. S., Kneib, J.-P., Richard, J., \& Kuijken, K. 2004, ApJ, 606,683

Schmidt, R. W., Allen, S. W., \& Fabian, A. C. 2001, MNRAS, 327, 1057

Smail, I., Ellis, R. S., Fitchett, M. J., Norgaard-Nielsen, H. U., Hansen, L., \& Jorgensen, H. E. 1991, MNRAS, 252, 19

Smail, I., Hogg, D. W., Blandford, R., Cohen, J. G., Edge, A. C., \& Djorgovski, S. G. 1995, MNRAS, 277, 1

Smail, I., Ivison, R. J., Blain, A. W., \& Kneib, J.-P. 2002, MNRAS, 331,495

Smith, G. P., Kneib, J.-P., Ebeling, H., Czoske, O., \& Smail, I. 2001, ApJ, 552,493

Smith, G. P., Kneib, J.-P., Smail, I., Mazzotta, P., Ebeling, H., \& Czoske, O. 2005, MNRAS, 359, 417

Smith, J. A., et al. 2002, AJ, 123, 2121

Spergel, D. N., et al. 2006, arXiv astro-ph/0603449

Struble, M. F., \& Rood, H. J. 1987, ApJS, 63, 543

Stoughton, C., et al. 2002, AJ, 123, 485

Tucker, D. L., et al. 2006, Astron. Nachr., 327, 821

Tyson, J. A., Kochanski, G. P., \& dell'Antonio, I. P. 1998, ApJ, 498, L107

Wambsganss, J., Bode, P., \& Ostriker, J. P. 2004, ApJ, 606, L93

Yee, H. K. C., Ellingson, E., Bechtold, J., Carlberg, R. G., \& Cuillandre, J.-C. 1996, AJ, 111, 1783

York, D. G., et al. 2000, AJ, 120, 1579

Zaritsky, D., \& Gonzalez, A. H. 2003, ApJ, 584, 691 